\title{
ACCELERATED AUTOMATION AND THE ECOSYSTEM OF ADAPTATION TO THE CHANGES
}

\section{QUJ BICHIA}

\author{
Doctor of Economics, Researcher \\ The Institute for the Research of Economic \\ and Social Problems of Globalization, \\ European University, Georgia \\ q.bichia@iset.ge
}

Abstract. The spread of the pandemic has further accelerated the already accelerated process of automation, as it reduces all interactions and helps social distancing, as it was advised to combat the virus. Automation is proceeding at a very fast pace in many sectors of the economy and creating the need for countries to respond to these new challenges at the level of national economic strategy. The new challenge has changed the attitudes of not only manufacturers but also consumers and given them additional impetus to the using robots and artificial intelligence. During this period, those, who are the first to be able to switch to online sales, reduce the need for communication with employees and increase the level of automation, will be able to gain an advantage. Because of this, there is a danger that after the pandemic ends, some of those currently temporarily unemployed will not be able to return to work and will need to adapt to the new reality. In the light of such changes, it is important to identify areas of declining and growing sectors of labor demand and to prepare the population for change to prevent technological unemployment. As it turns out, technological skills and social skills are becoming increasingly demanded, while physical and basic cognitive skills are on the decline. In order to adapt to a new reality, firms have a choice of retraining old employees, redeploying them to different sets of tasks, hiring individuals with skills that are increasingly needed, contracting specialists outside the company (freelancers) and releasing employees that do not fit the new paradigm of business models.

Automation involves the disappearance of a large proportion of low-skilled occupations, leading to a critical role for education in employment. It is therefore important for the government to establish a strategic position on higher and professional education, which will be based on a long-term plan for the structural transformation of the workforce. Automation may be used as an opportunity if a productive ecosystem is established and all stakeholders get actively involved in the process of adjusting to the new reality. For that it is necessary to form an overall ecosystem of stakeholders, which should contain employers at the center and different supporting institutions around them. The ecosystem should consist of labor agencies that help individuals find new jobs, retrain and get some benefits. Educational institutions play a crucial role in devising courses and specializations that fit an exact position that is in demand in some companies. Industry associations and non-profit organizations also need to be coordinated with companies to expand this ecosystem. Combined effort of all these five groups of stakeholders can manage to overcome the looming challenge of rapid unemployment growth and wide skill mismatch. If a society fail to coordinate in the ecosystem, the aftermath will be devastating and income inequality will be amplified. One solution to that is thought to be universal basic income (UBI) but so far it doesn't seem feasible and cannot be relied on. Adapting society to a change instead of giving out basic income seems to address the problem better and not just on a surface.

KEYWORDS: FOURTH INDUSTRIAL REVOLUTION, AUTOMATION, TECHNOLOGICAL UNEMPLOYMENT, LABOR AGENCIES.

For citation: Bichia, Q., (2020). Accelerated Automation and the Ecosystem of Adaption to the Changes. Globalization and Business, 10. 233-242. (In Georgian) https://doi.org/10.35945/gb.2020.10.030 


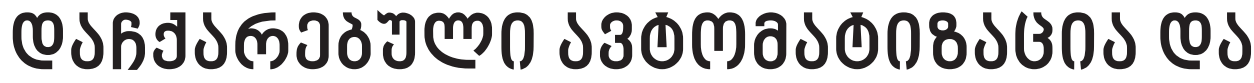

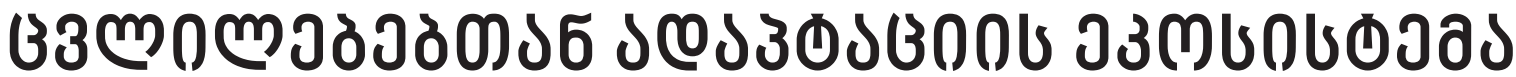

\section{รગち0 ठ0ङ0১}

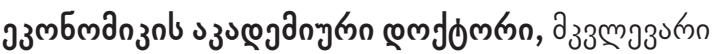

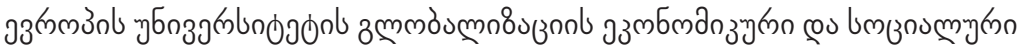

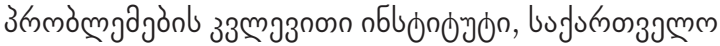

q.bichia@iset.ge

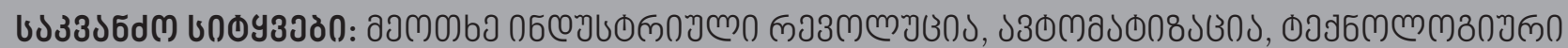

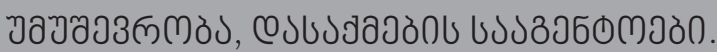

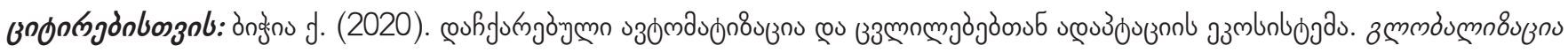
so don86jbn, №10, 33. 233-242. https://doi.org/10.35945/gb.2020.10.030

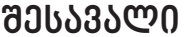

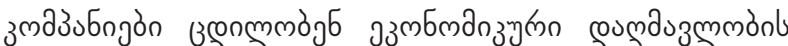

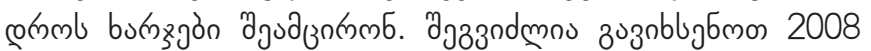

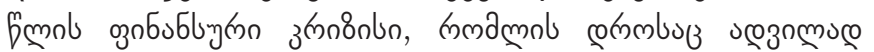

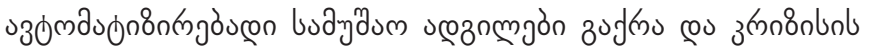

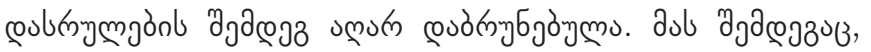

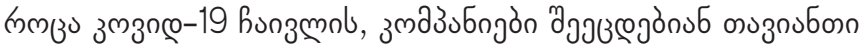

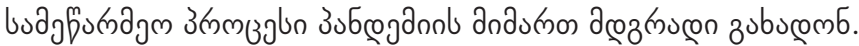

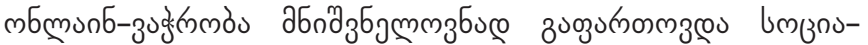

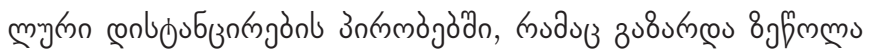

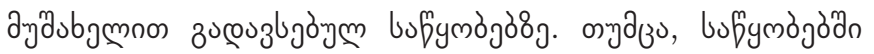

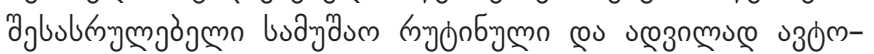

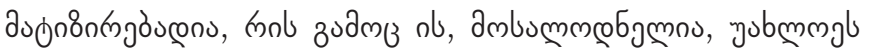

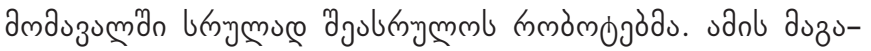

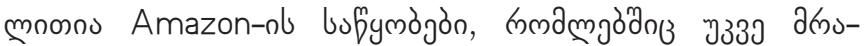

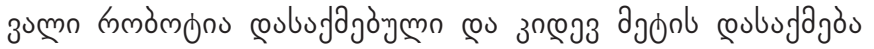

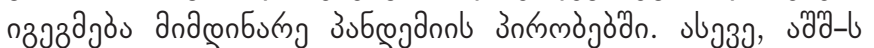

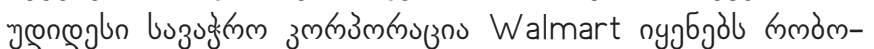

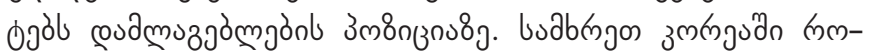

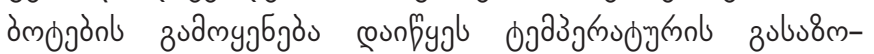

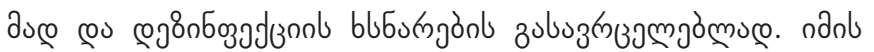

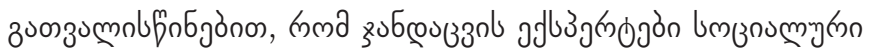

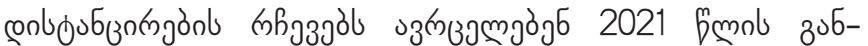

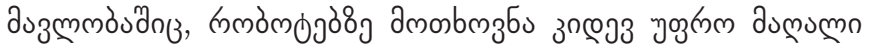

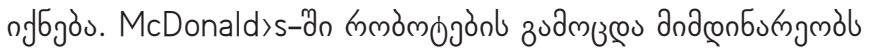

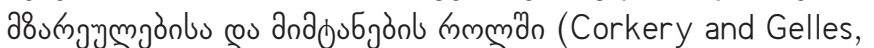

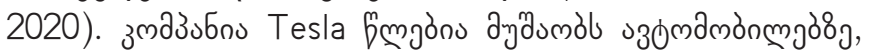

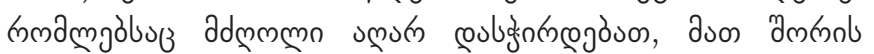

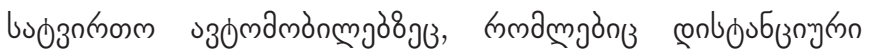

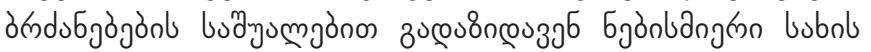

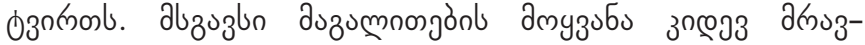

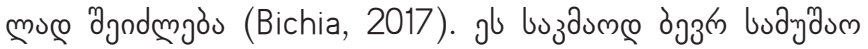

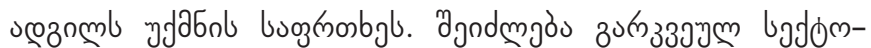

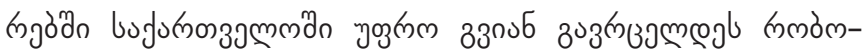

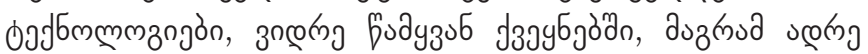

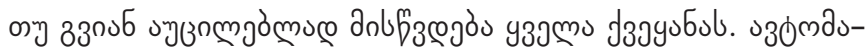

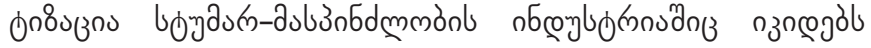

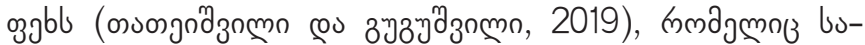

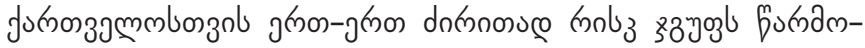

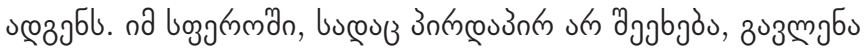

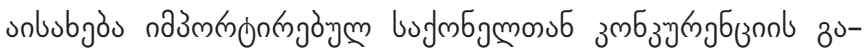

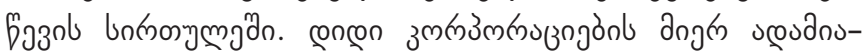

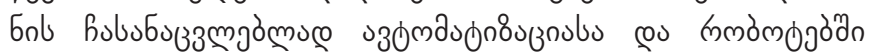

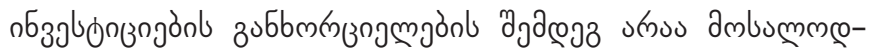

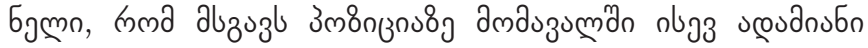

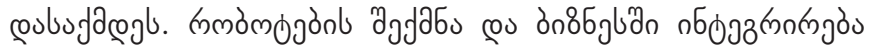

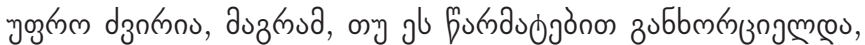

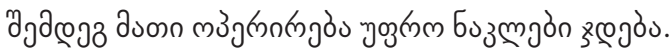

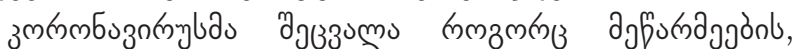

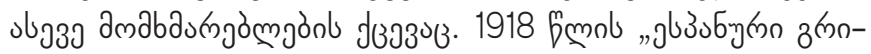

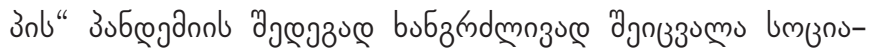

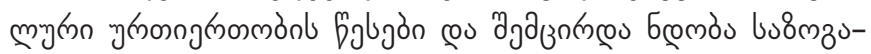

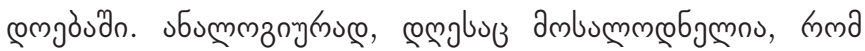

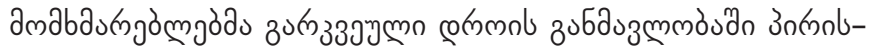

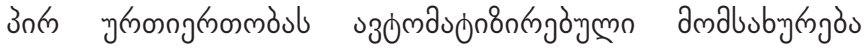

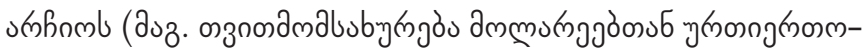

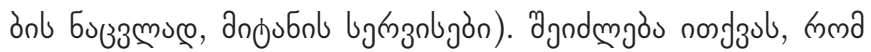

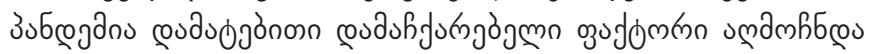

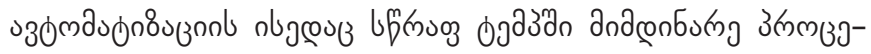

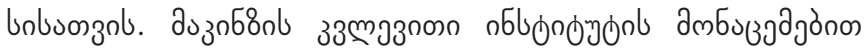

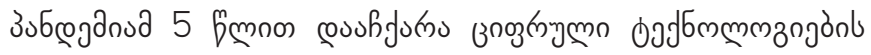




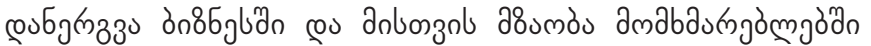

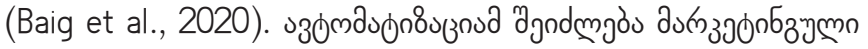

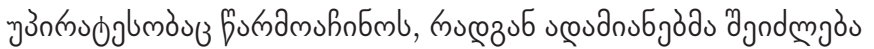

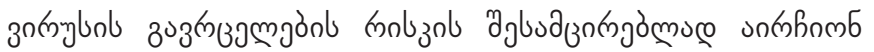

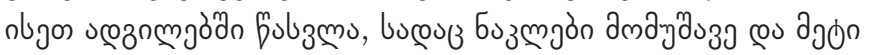

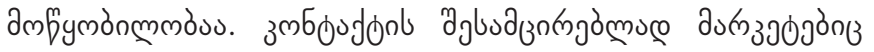

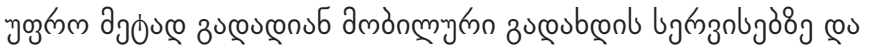

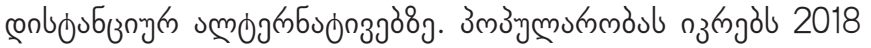
fosmb cous

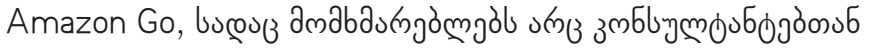

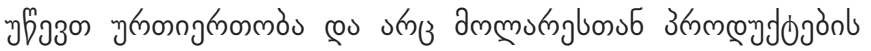
300uknjos.

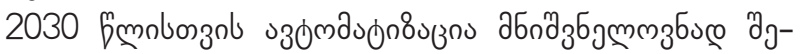

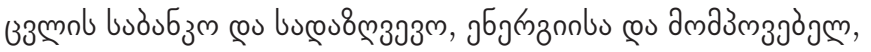

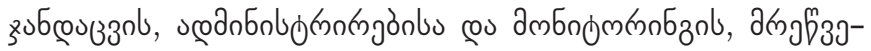

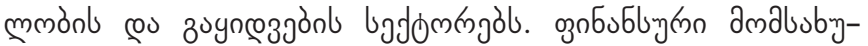

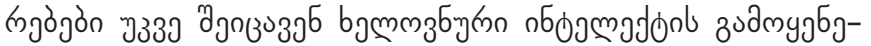

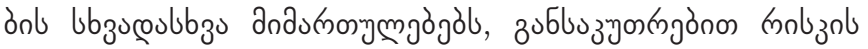

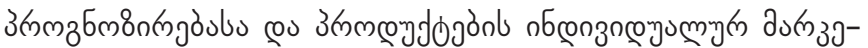

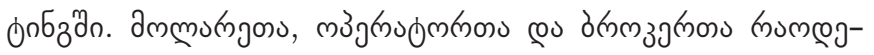

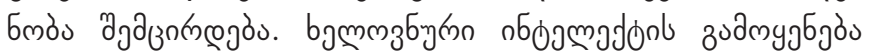

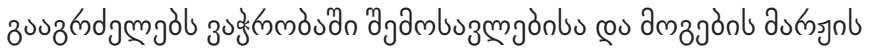

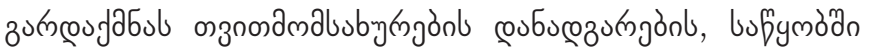

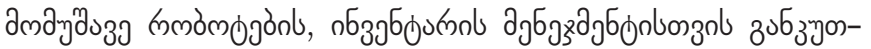

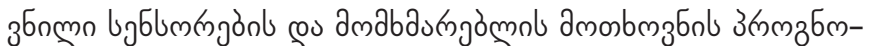

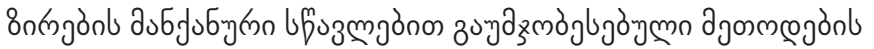

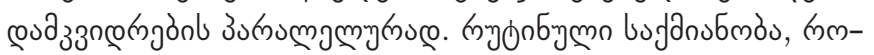

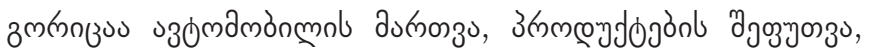

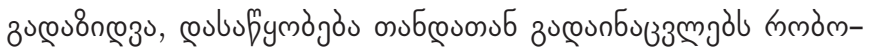

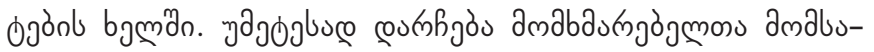

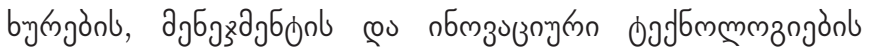

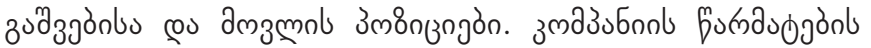
dnగnnosucon zubabu\&

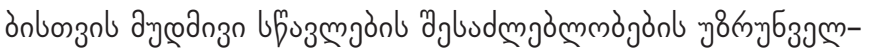

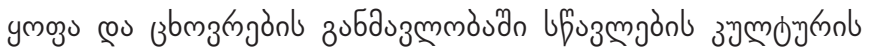

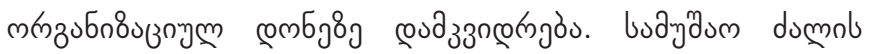

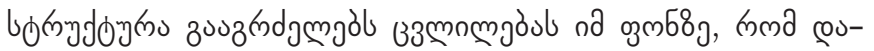

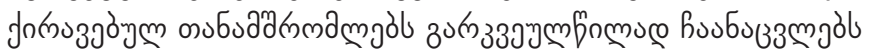

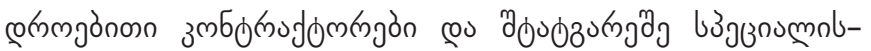

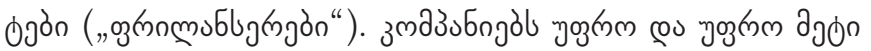

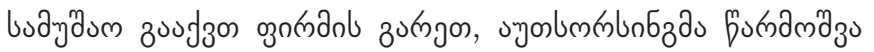

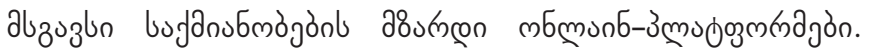

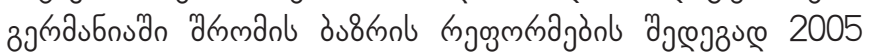

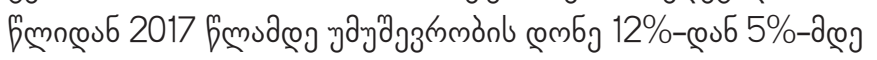

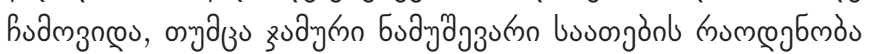

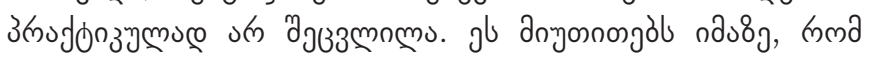

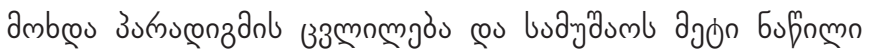

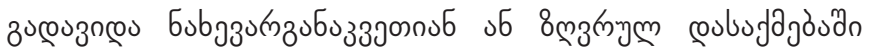

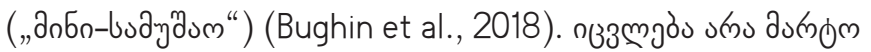

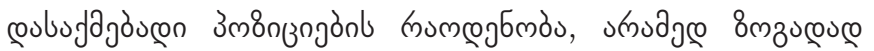

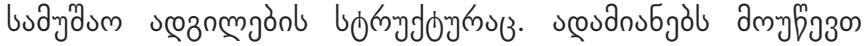

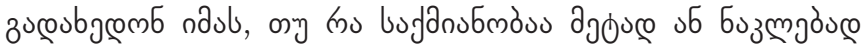

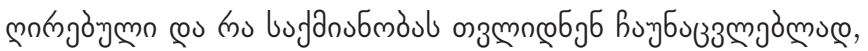

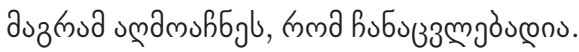

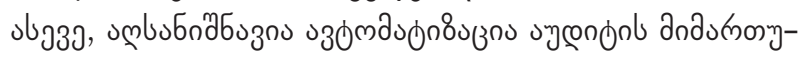

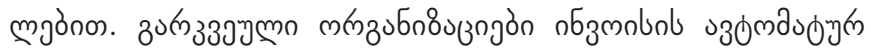

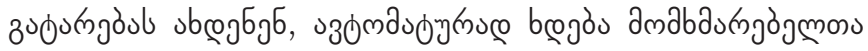

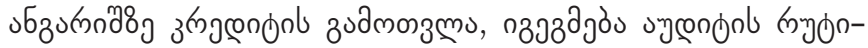

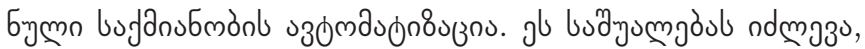

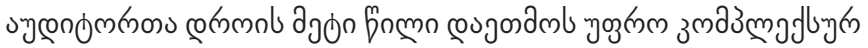

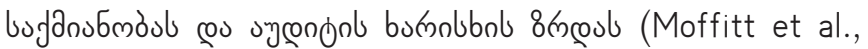
2018).

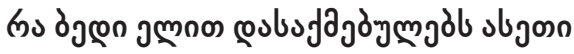

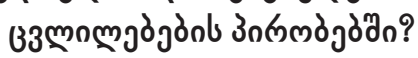

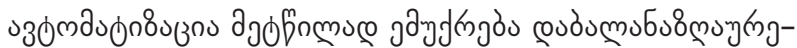

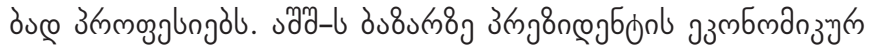

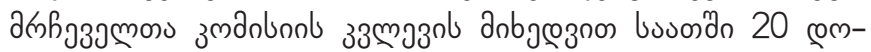

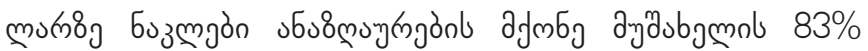

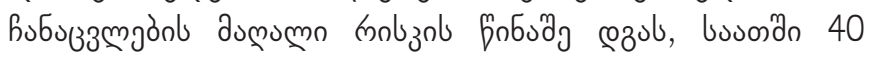

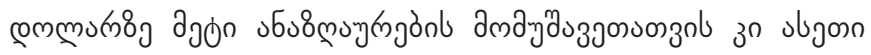

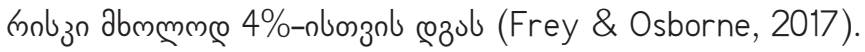

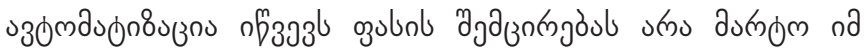

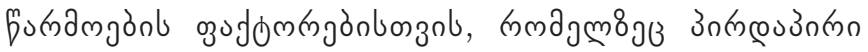

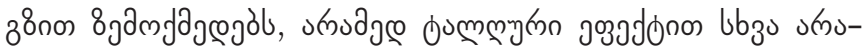

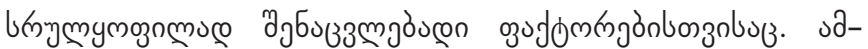

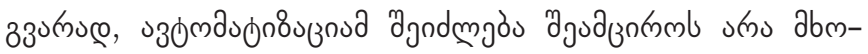

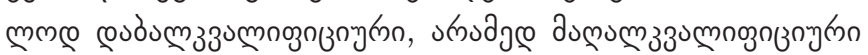

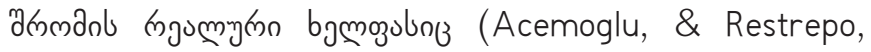

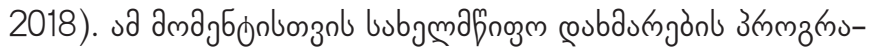

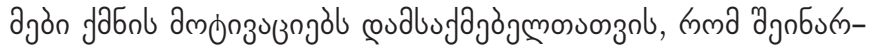

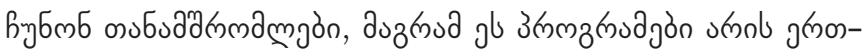

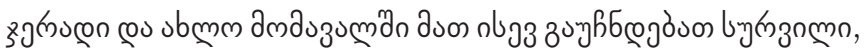

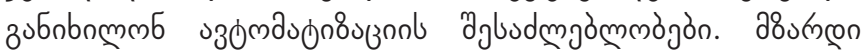

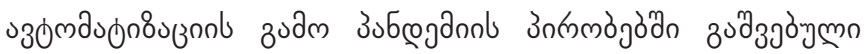

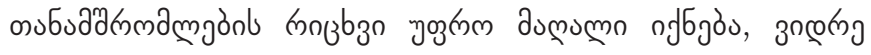

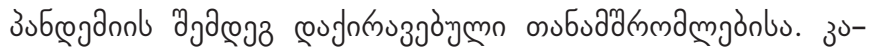

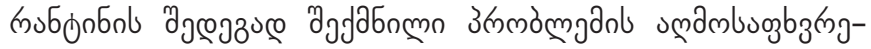

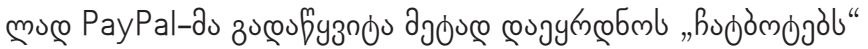

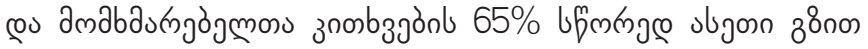

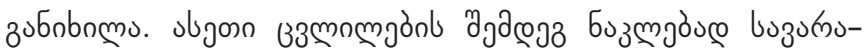

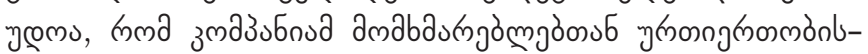

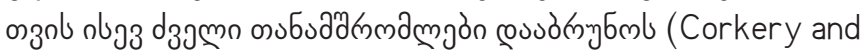

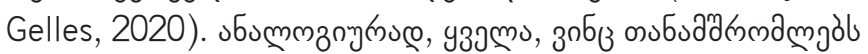

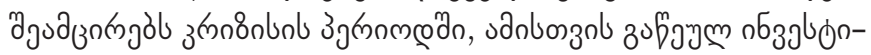

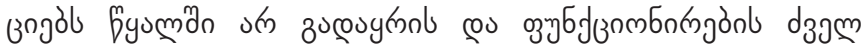

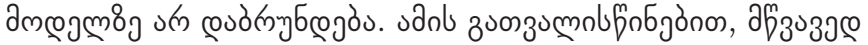

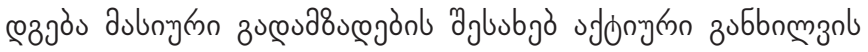
buznombn. 


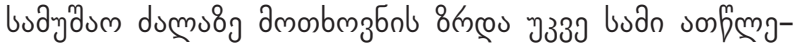

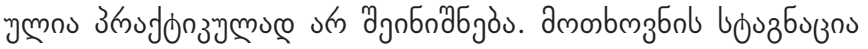

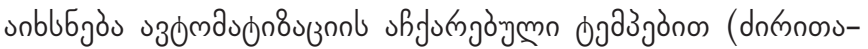
cose д๘

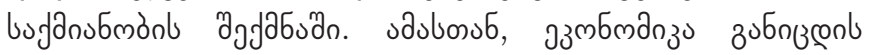

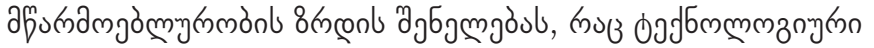

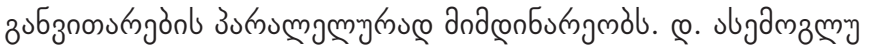

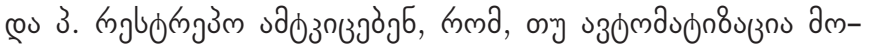

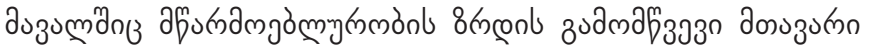

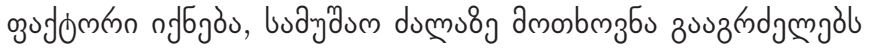

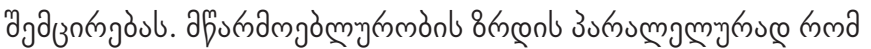

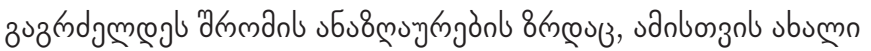

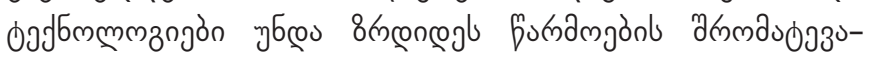

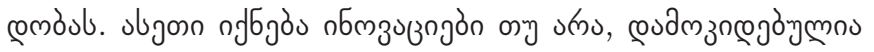

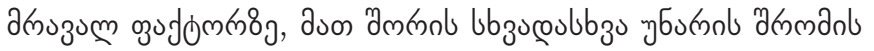
дпfмщ

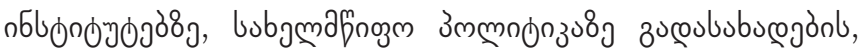

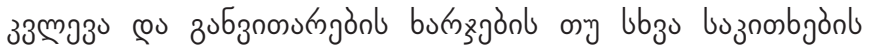

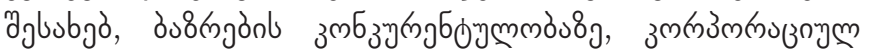

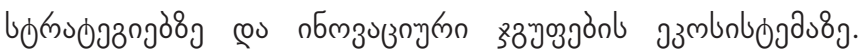

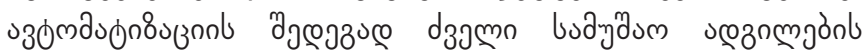

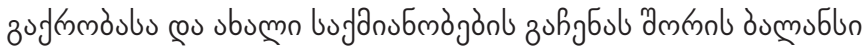

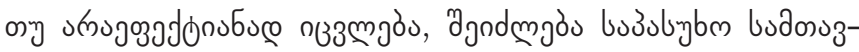

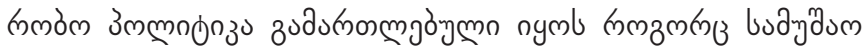
ง

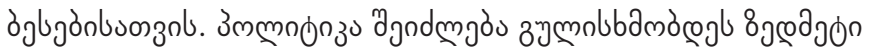

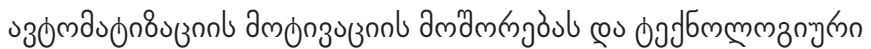

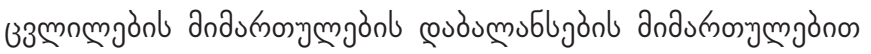

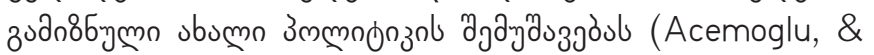
Restrepo, 2019).

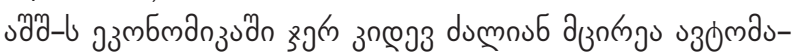

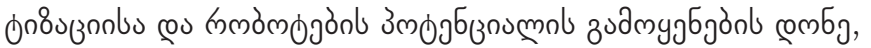

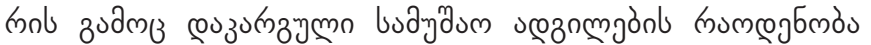

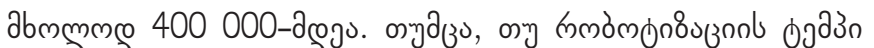

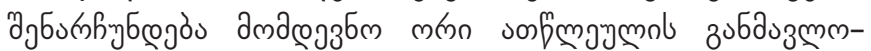

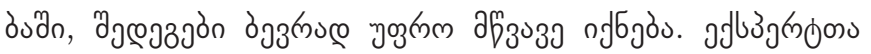

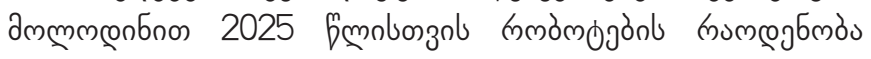

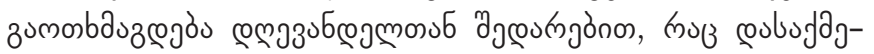

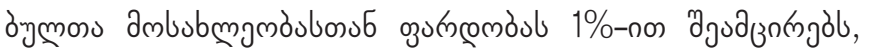

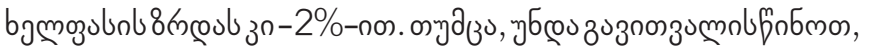

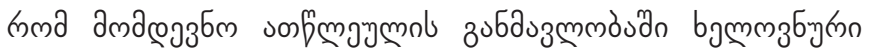

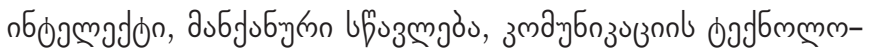

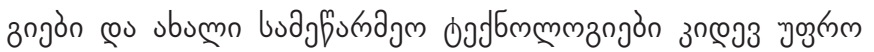

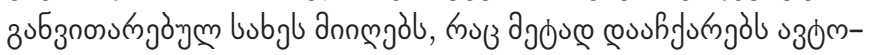

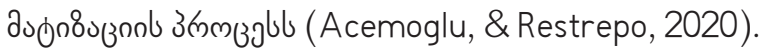

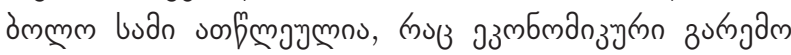

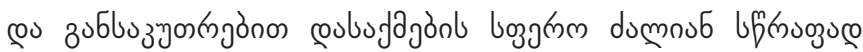

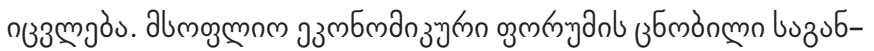

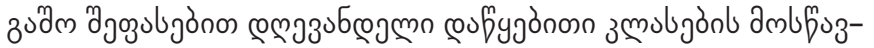

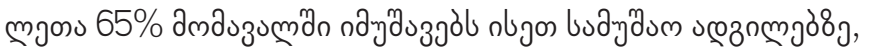

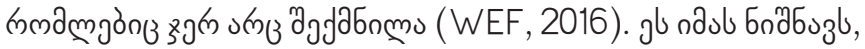

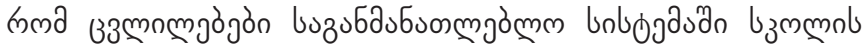

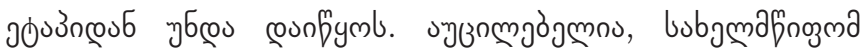

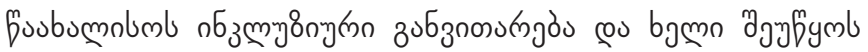

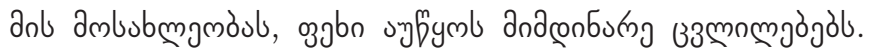

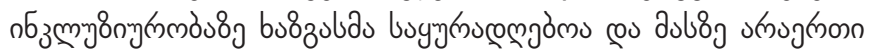

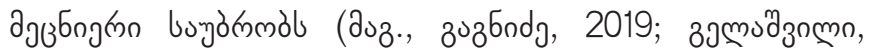

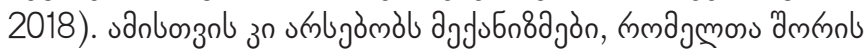

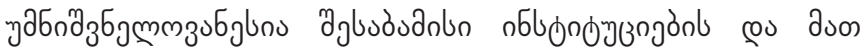

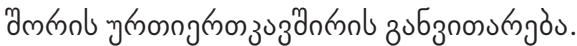

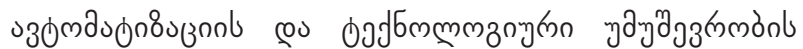
gुm68; buzucoububucom unbogas agndmgas zucoububgen

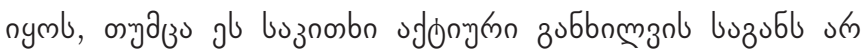

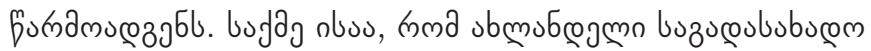

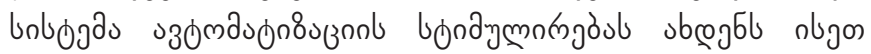

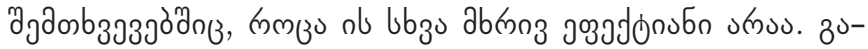

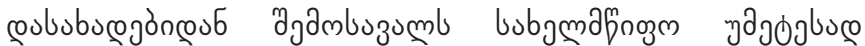

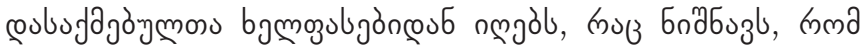

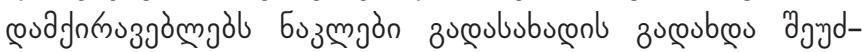

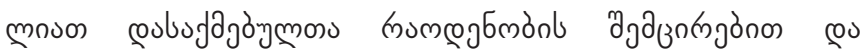

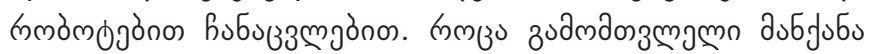

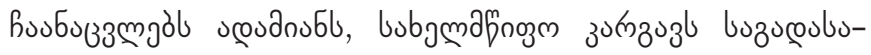

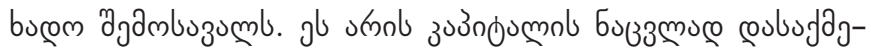

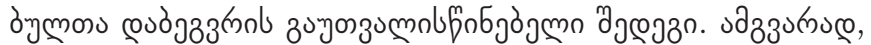

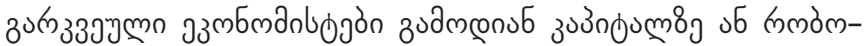

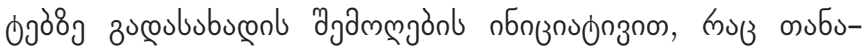

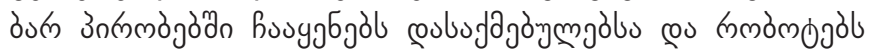
(Abbott \& Bogenschneider, 2018). gl bunбog

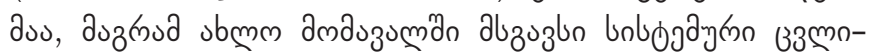

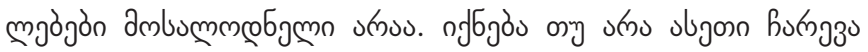

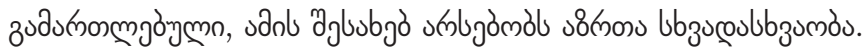

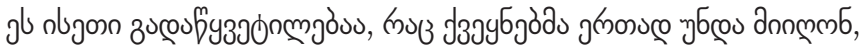

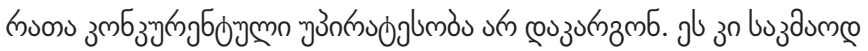

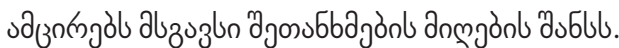

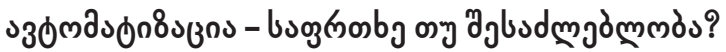

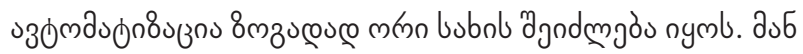

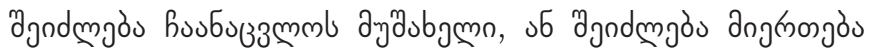

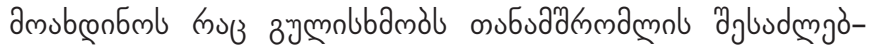

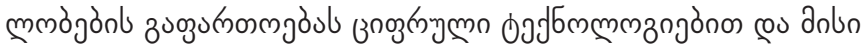

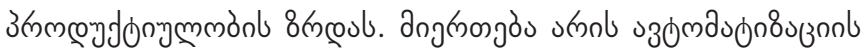

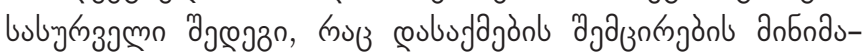

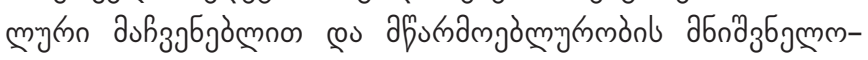

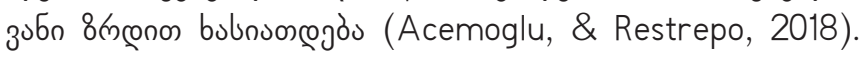

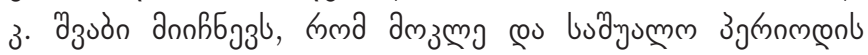

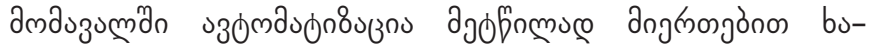

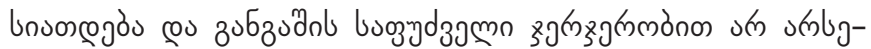

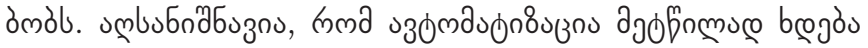
змбзб 


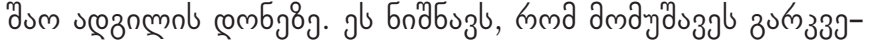

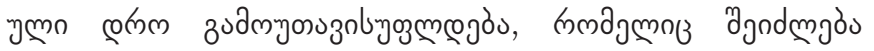

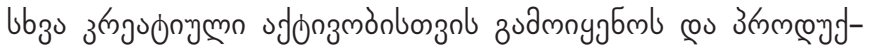
оп"ymm

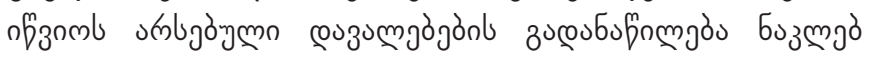

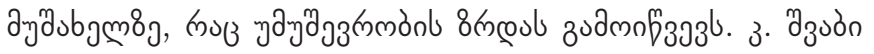

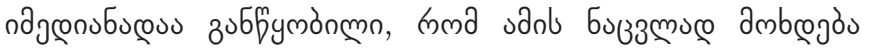

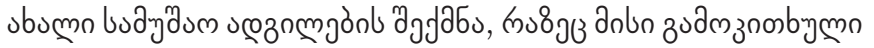

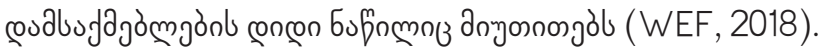

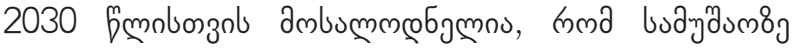

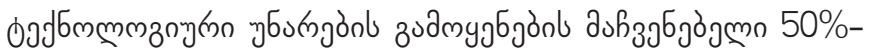

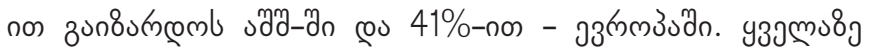

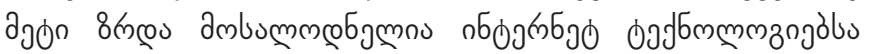

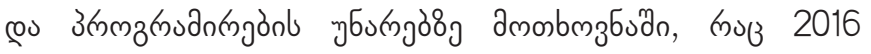

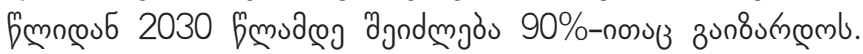

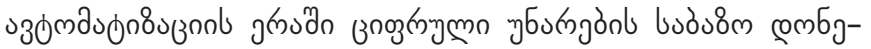

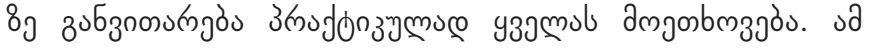

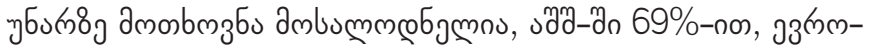

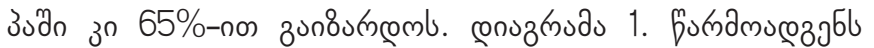

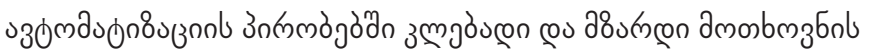
चб

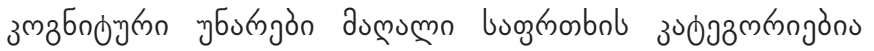

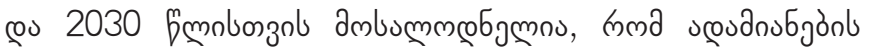

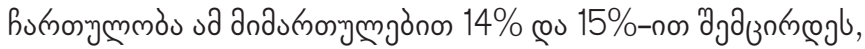

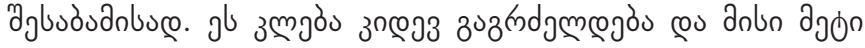

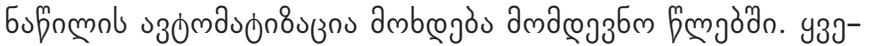

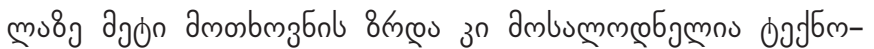

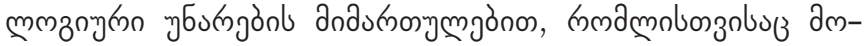

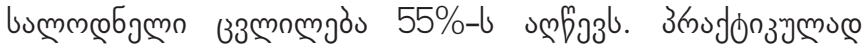

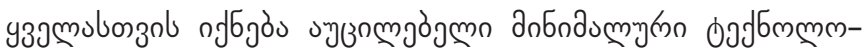

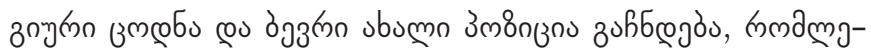

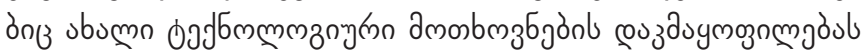
amgabubyrgyods (Bughin et al., 2018).

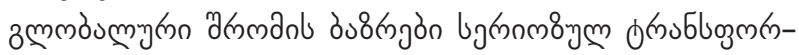

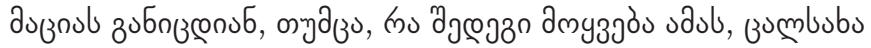

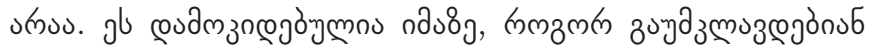

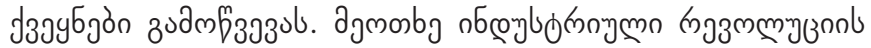

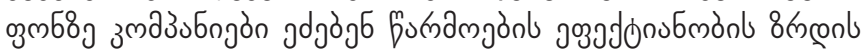

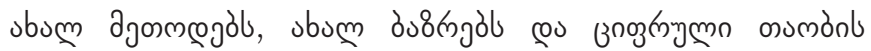

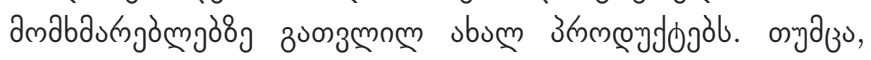

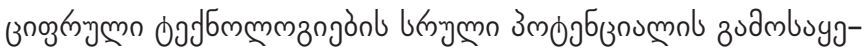

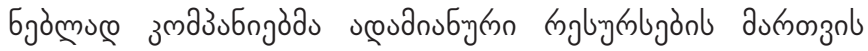

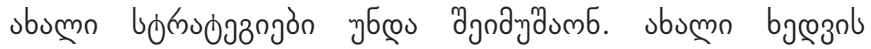

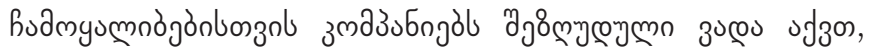
ஙоœ

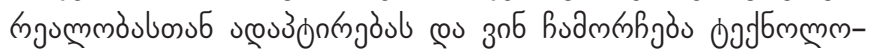

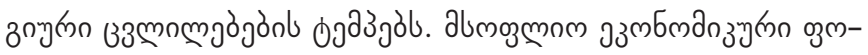

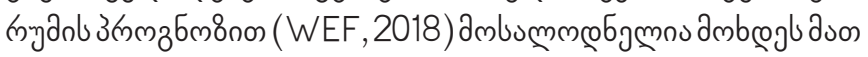

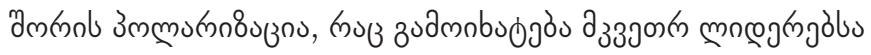

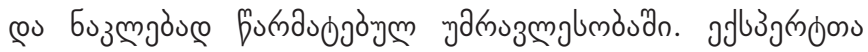

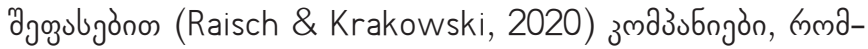
mjönz bymma

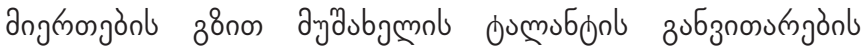

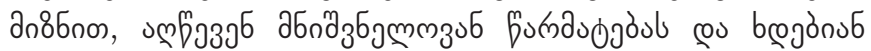

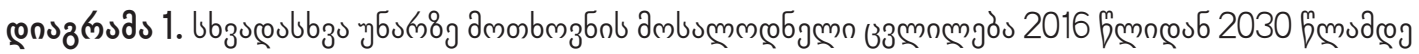

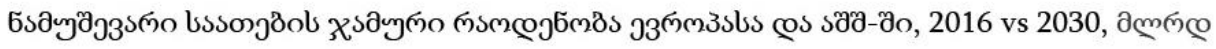

$2016 \bigcirc 2030$

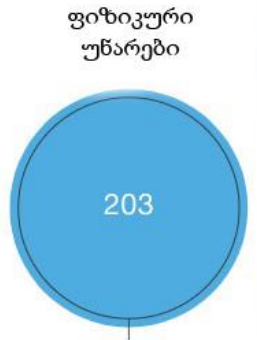

174

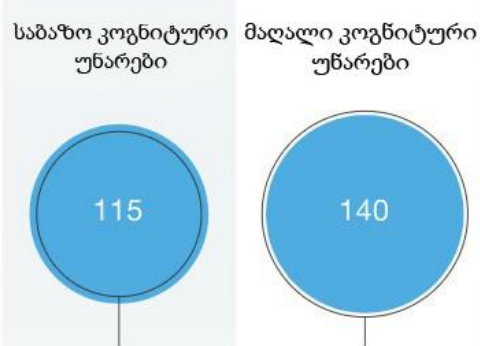

97

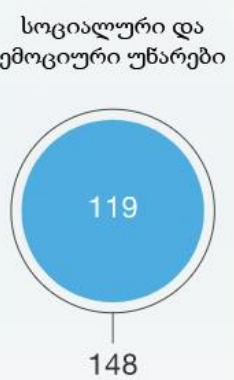

148

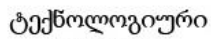

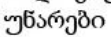

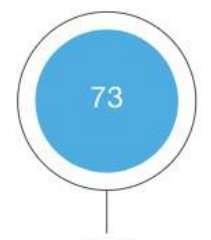

113

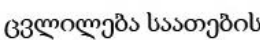

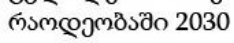

fimolonsol, \%

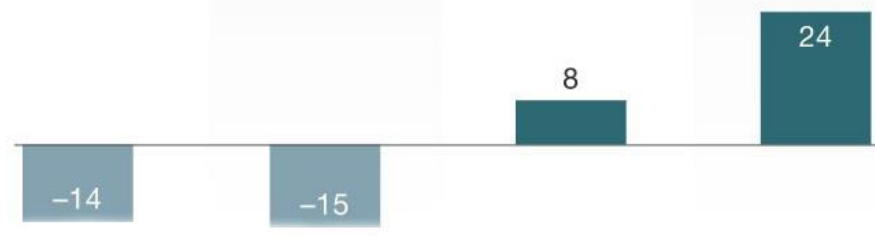

fyuかn: McKinsey Global Institute (2018) 


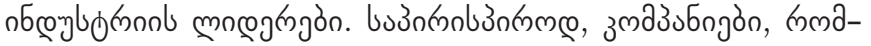

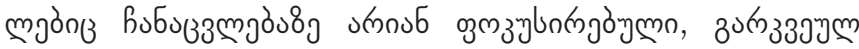

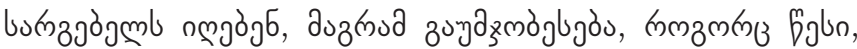

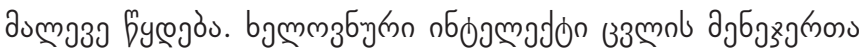

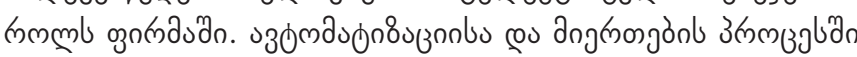

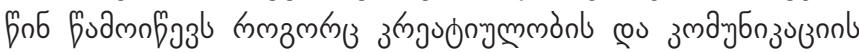

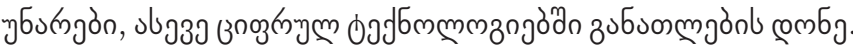

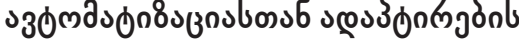 эзmbnbojas}

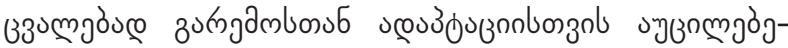

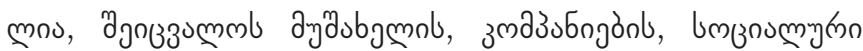

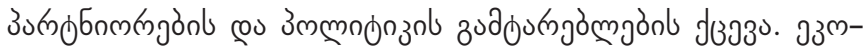

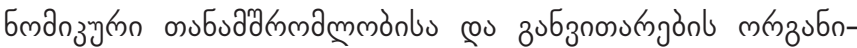

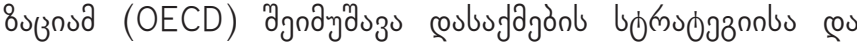

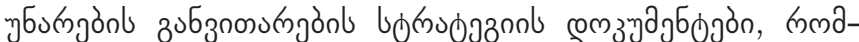

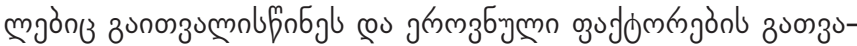

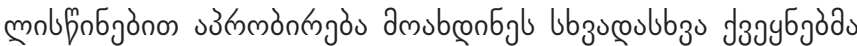

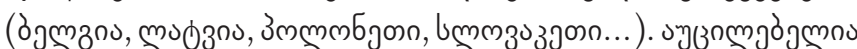

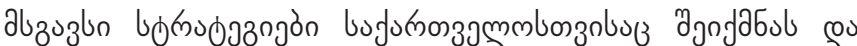

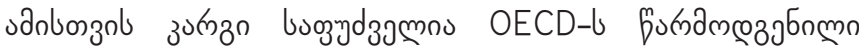

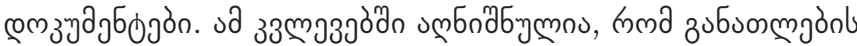

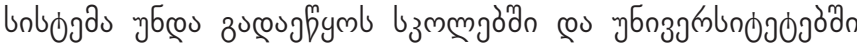

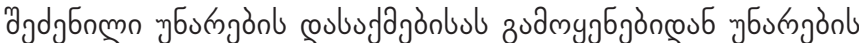

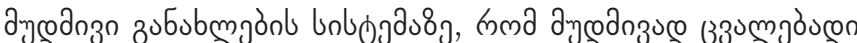

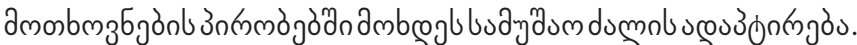

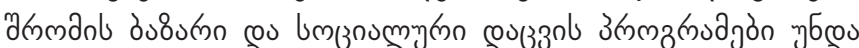

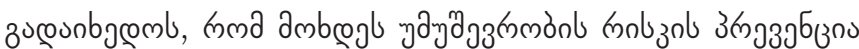

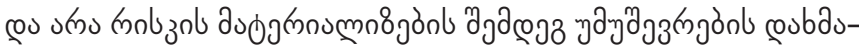

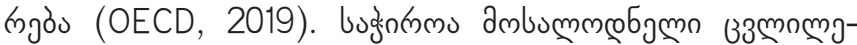

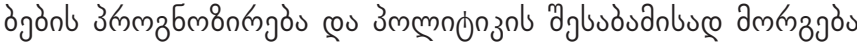

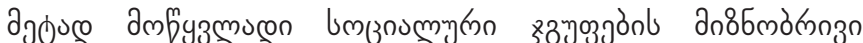

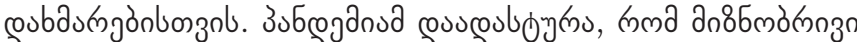

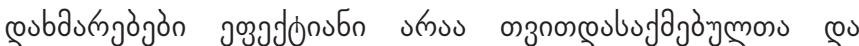

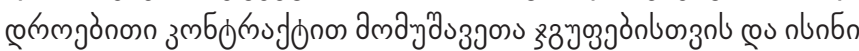

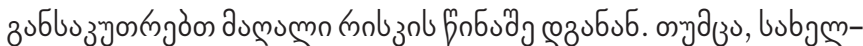

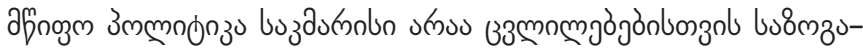

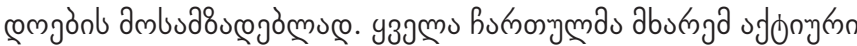

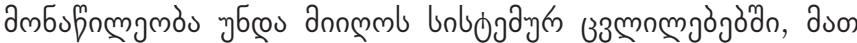

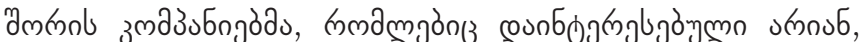

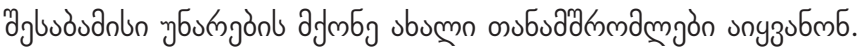

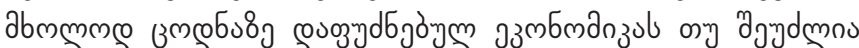

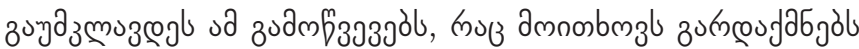

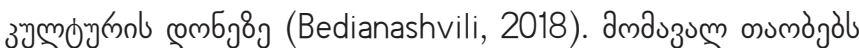

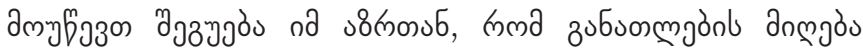

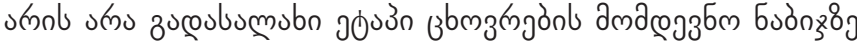

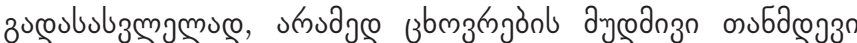

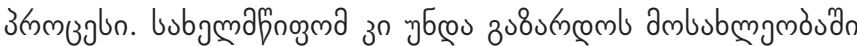

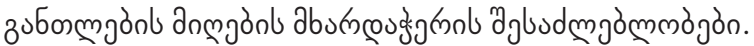

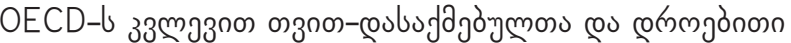

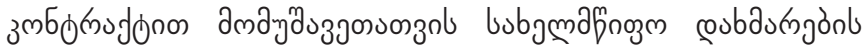

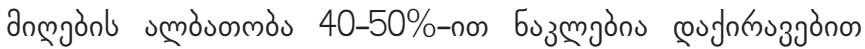

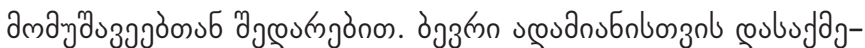

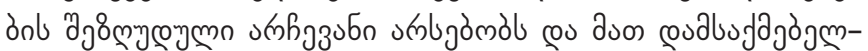

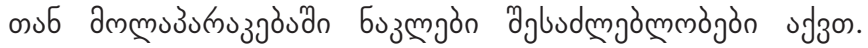

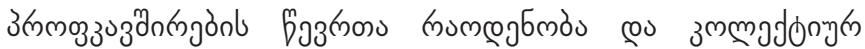

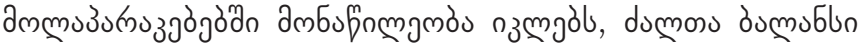

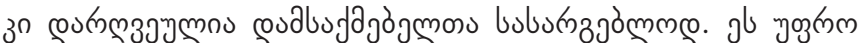

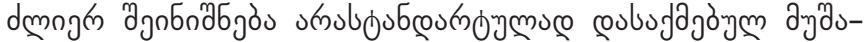

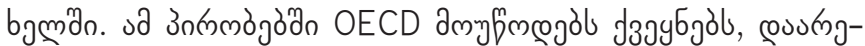

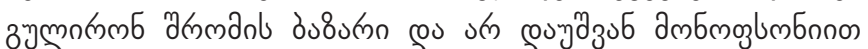

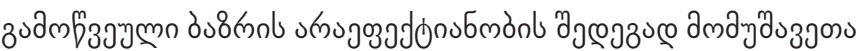

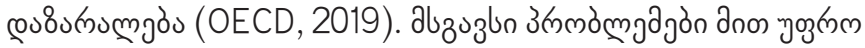

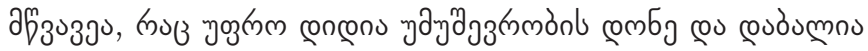

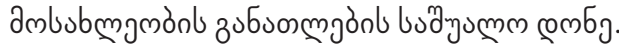

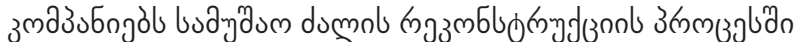

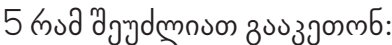

- ascosarcojós;

- avcouanbuavrnosgos;

- coujorrozgó,

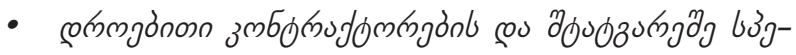

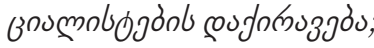

- zuosuznlyggrmgos (Bughin et al., 2018).

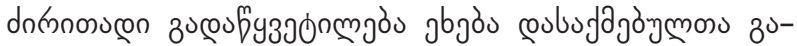

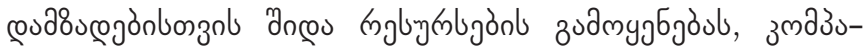

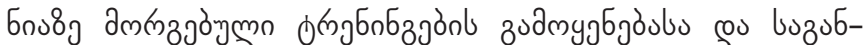

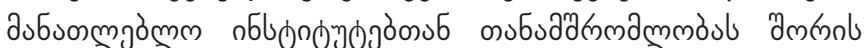

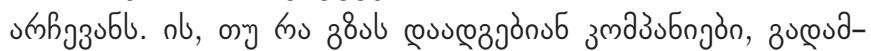

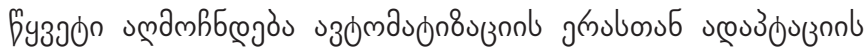

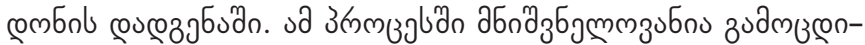

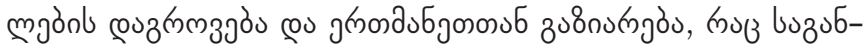

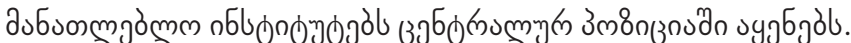

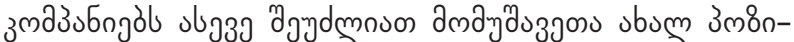

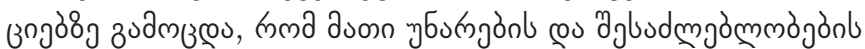

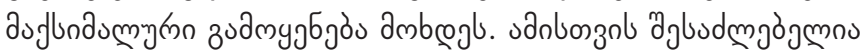
зuбufßnm

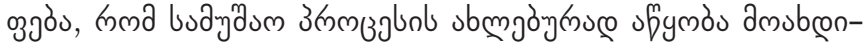

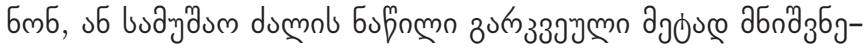

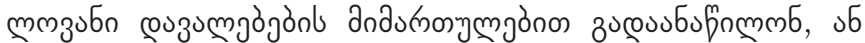

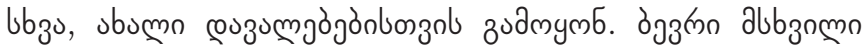

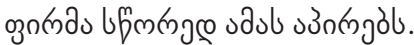

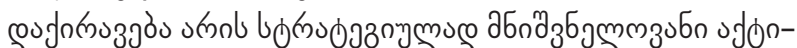

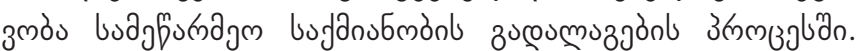

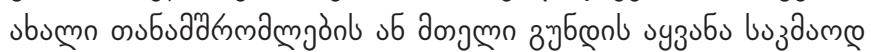

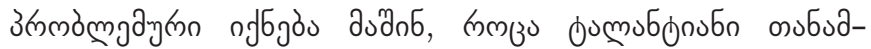

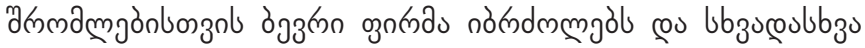

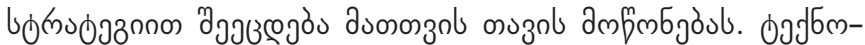

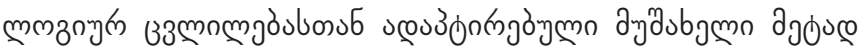

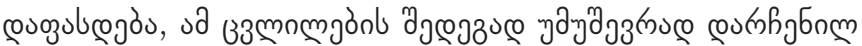




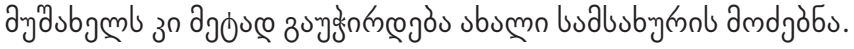

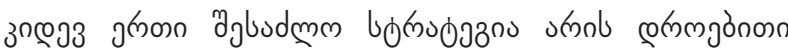

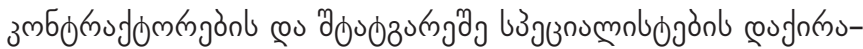

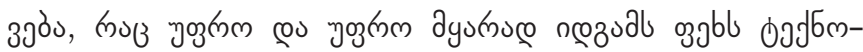

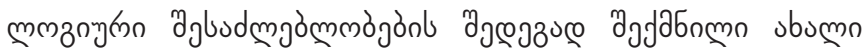

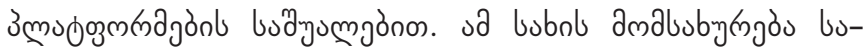

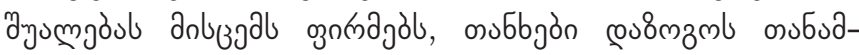

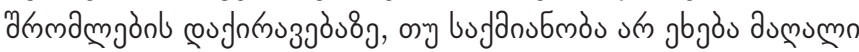

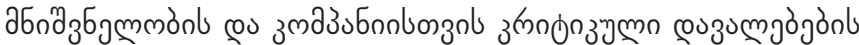

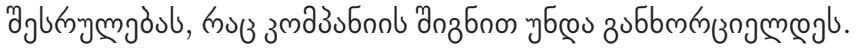

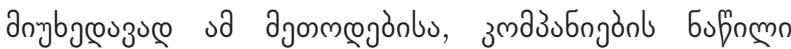

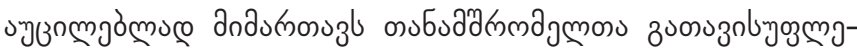

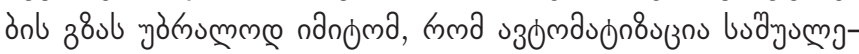

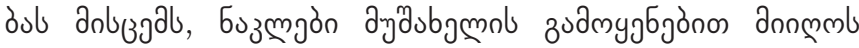

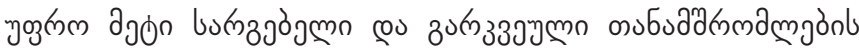

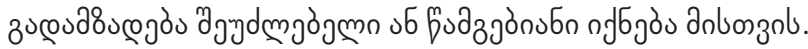

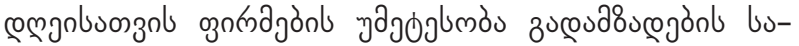

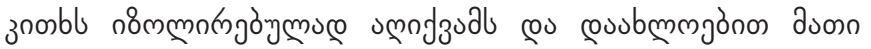

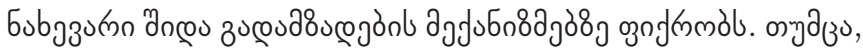

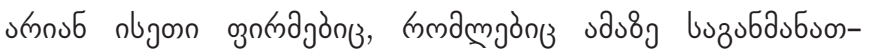

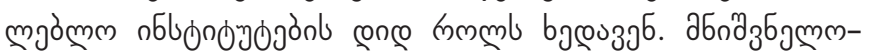

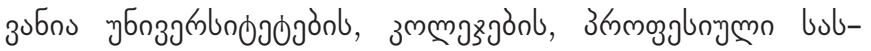

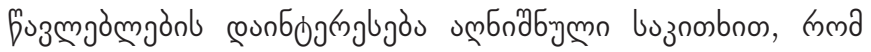

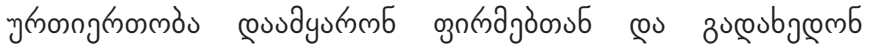

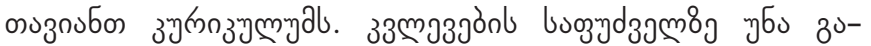

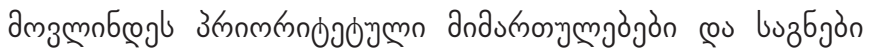

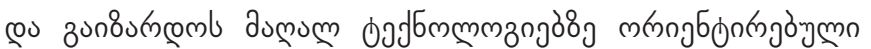

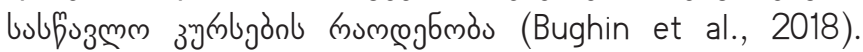

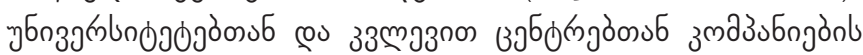

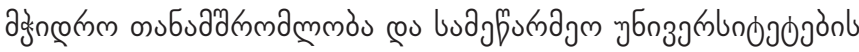

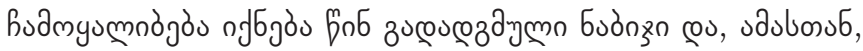

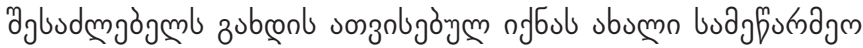

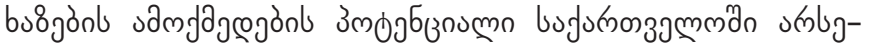

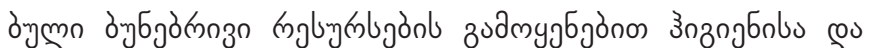

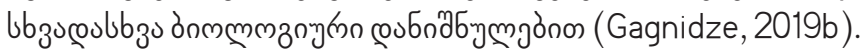

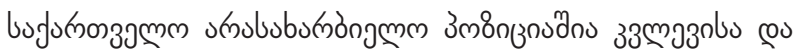

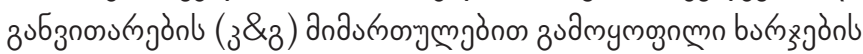

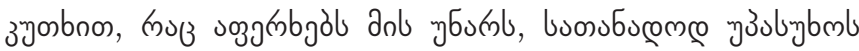

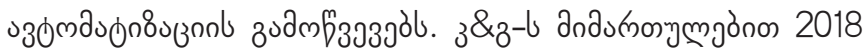

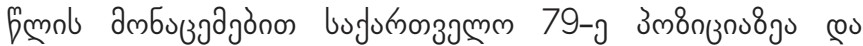

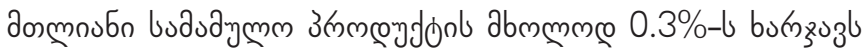

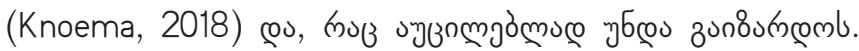

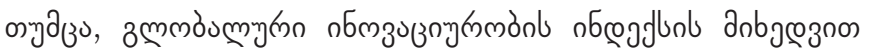
кradegr

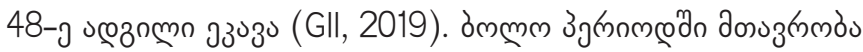

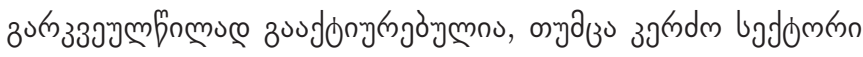

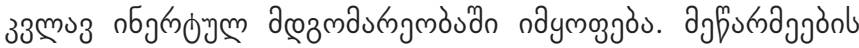

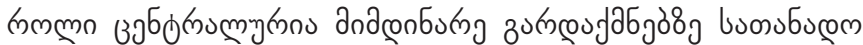

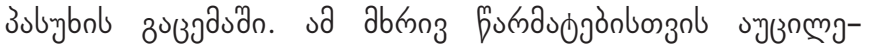

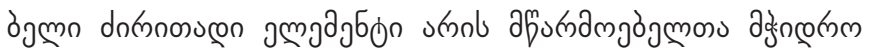

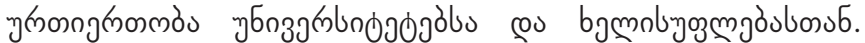

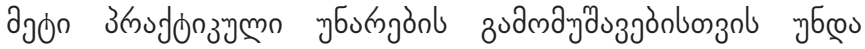

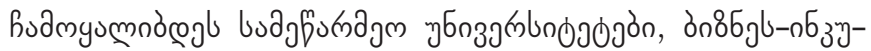

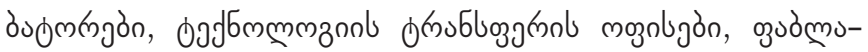

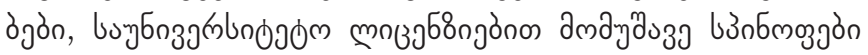

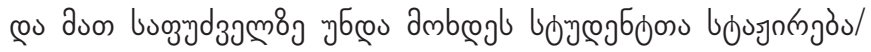

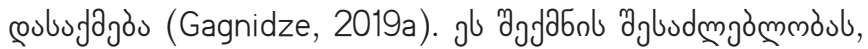

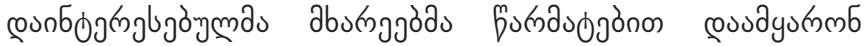

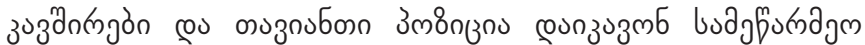

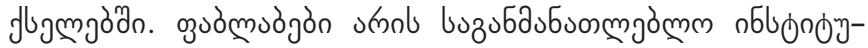

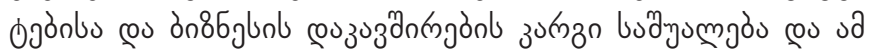

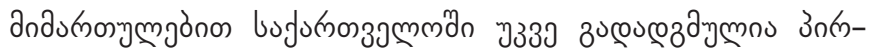

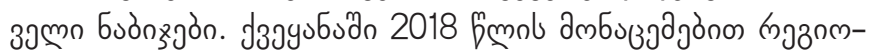

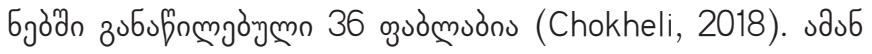

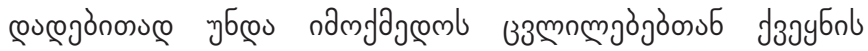

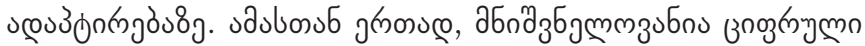

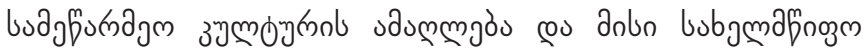

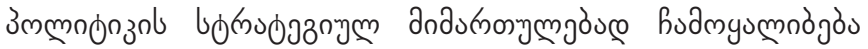

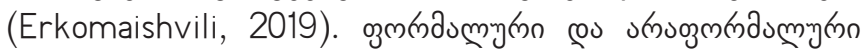

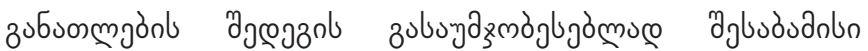

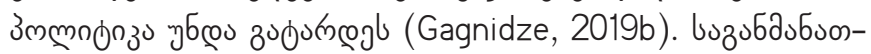

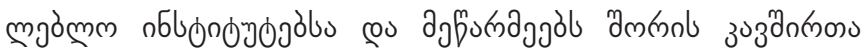

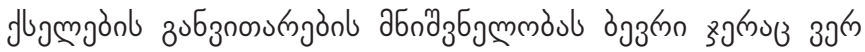

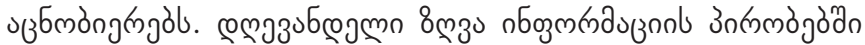

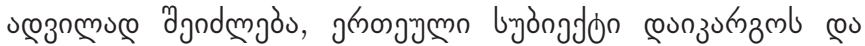

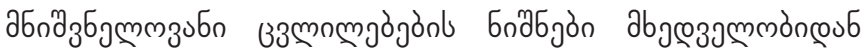

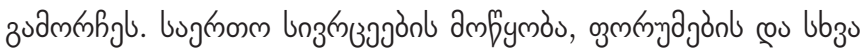

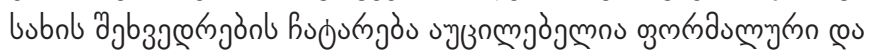

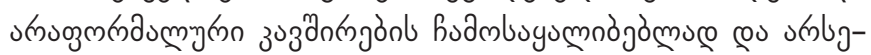

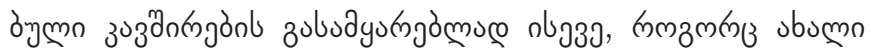

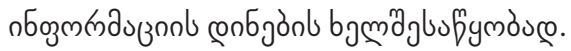

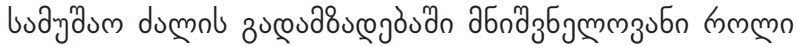

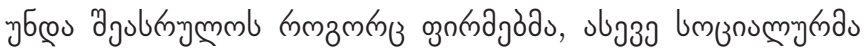

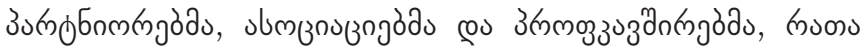

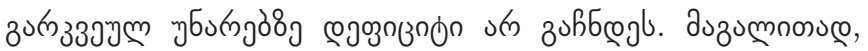

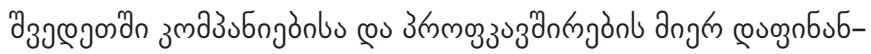

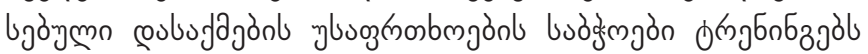

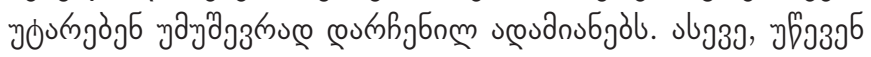

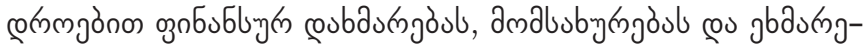

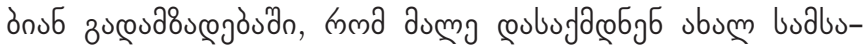

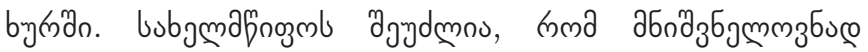

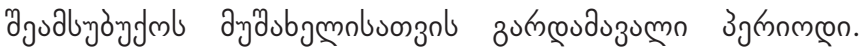

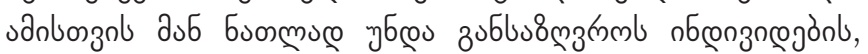

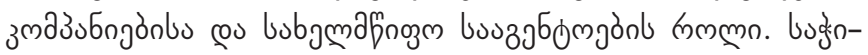

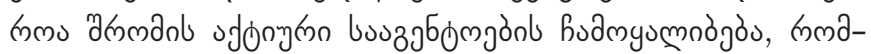

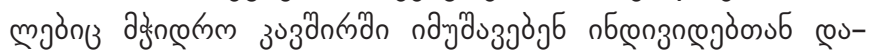

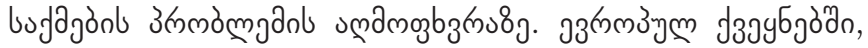

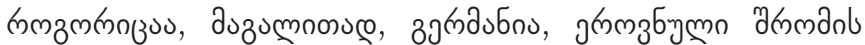

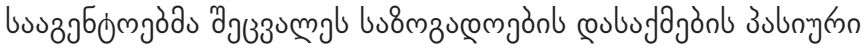

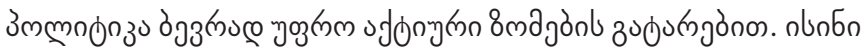




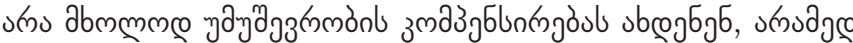

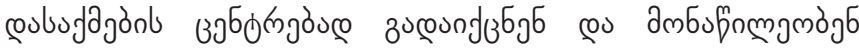

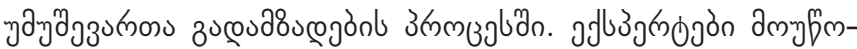

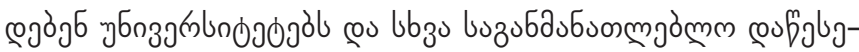

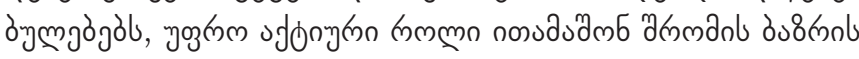

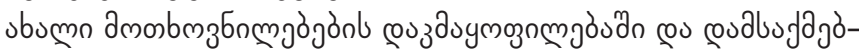

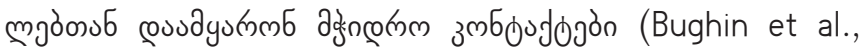

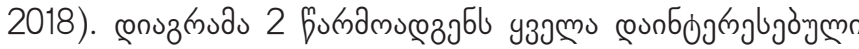

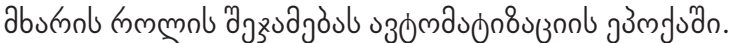

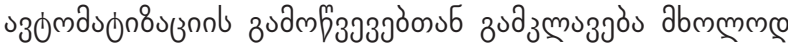

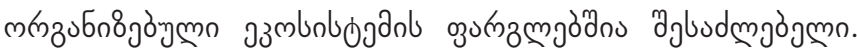

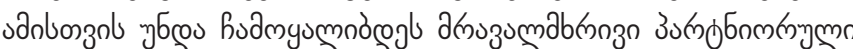

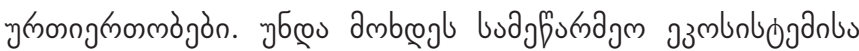

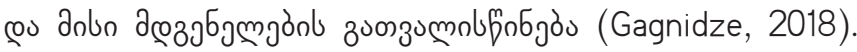

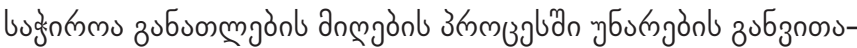

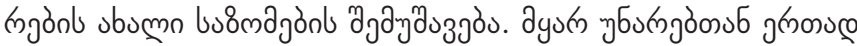

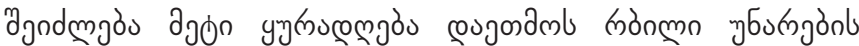

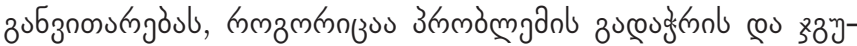

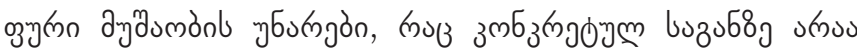

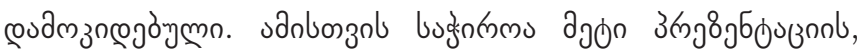

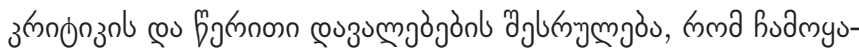

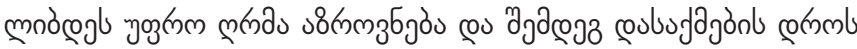

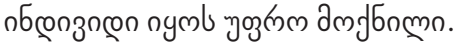

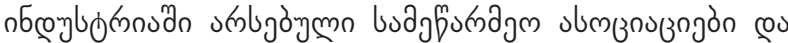

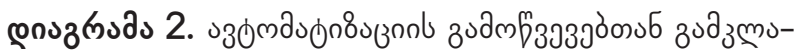
zjorn azmbnlogas

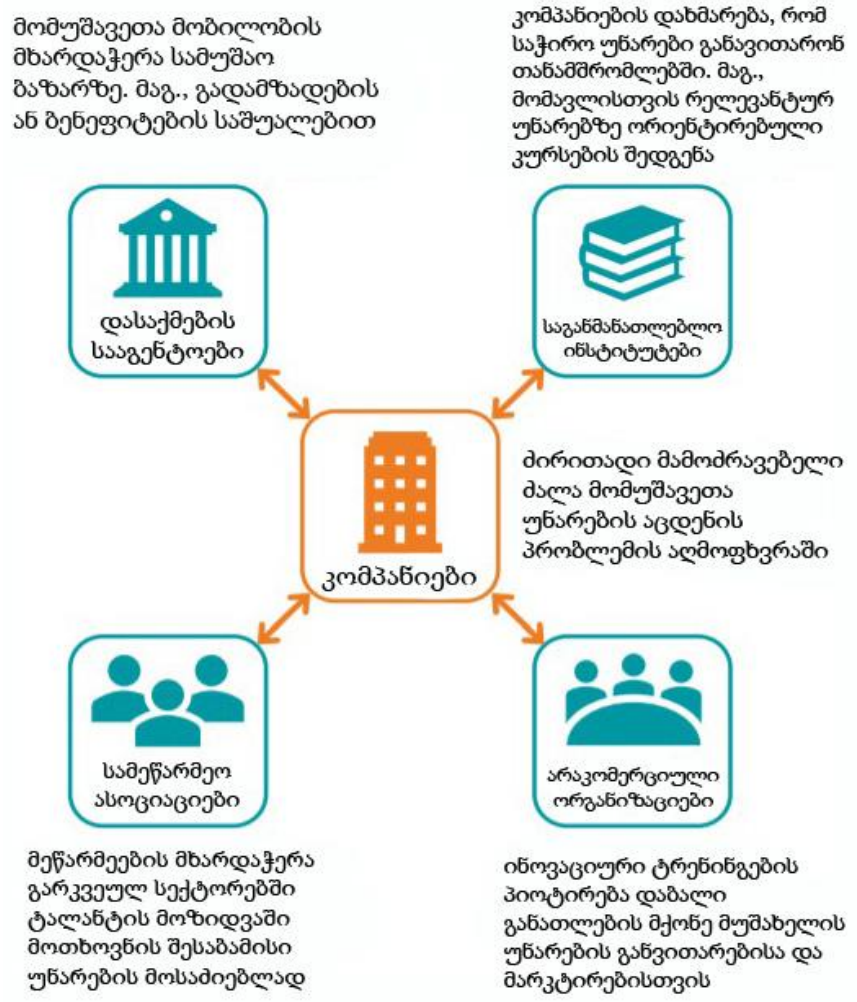

fyurnm: McKinsey Global Institute (2018)

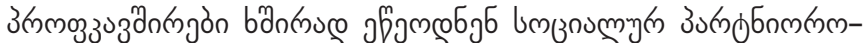

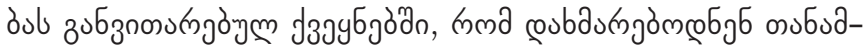

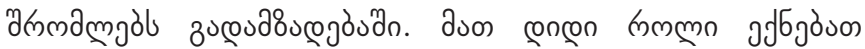

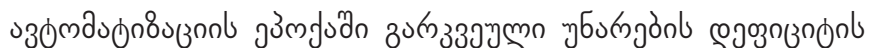

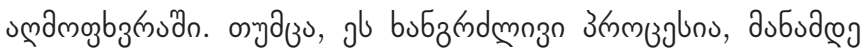

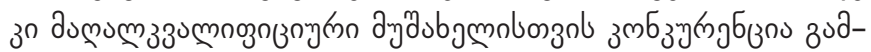

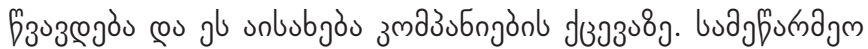

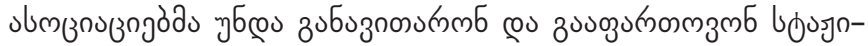

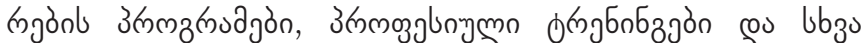

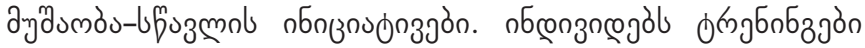

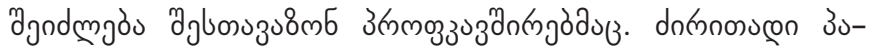

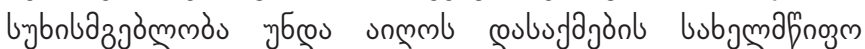

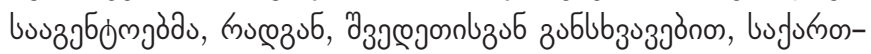

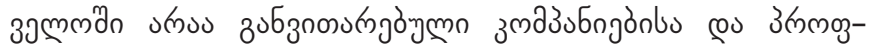
उз3

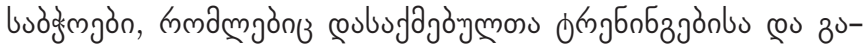

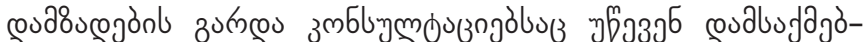

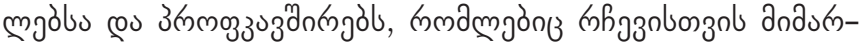

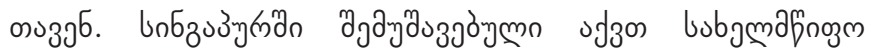

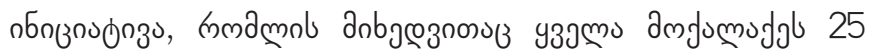

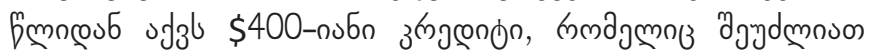

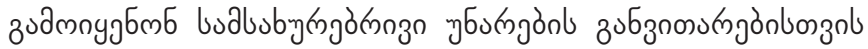

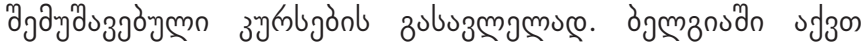

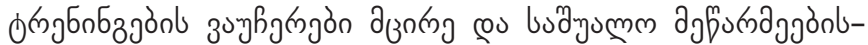

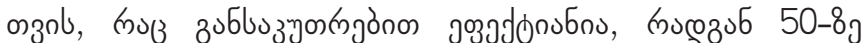

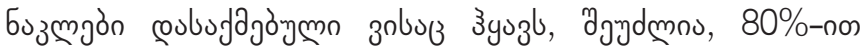

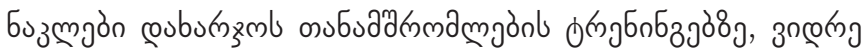

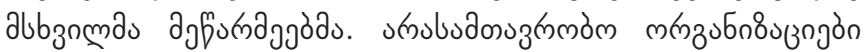

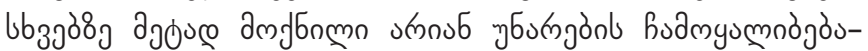

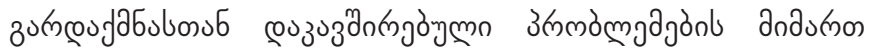

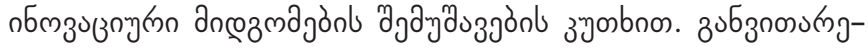

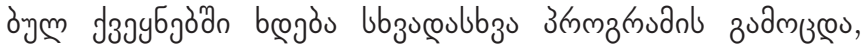

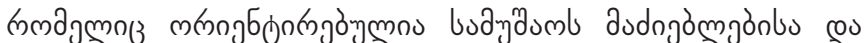

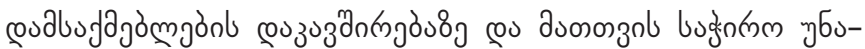

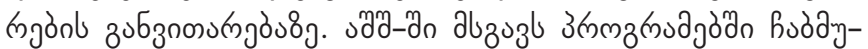

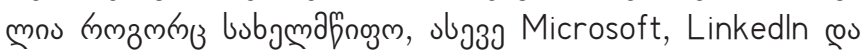

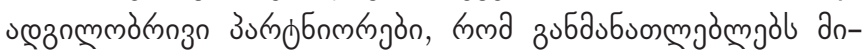

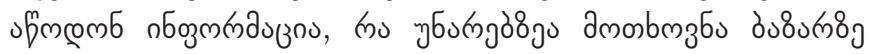

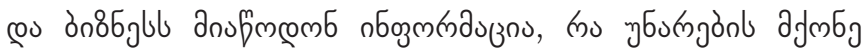

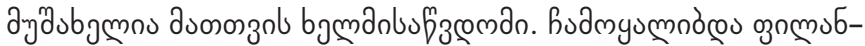

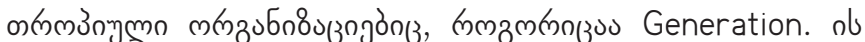
fз

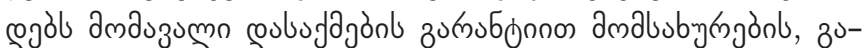

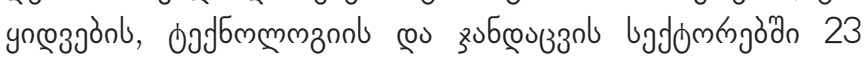

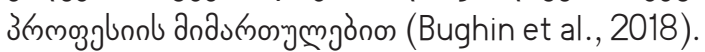

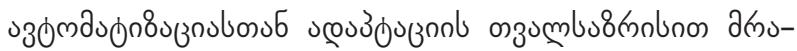

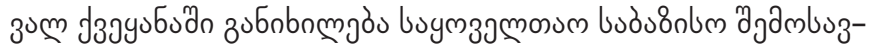

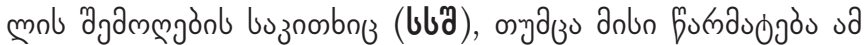

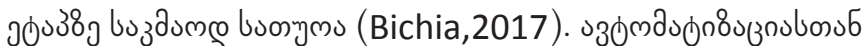

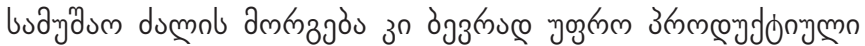




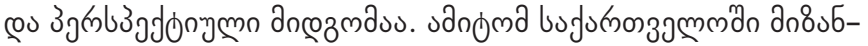

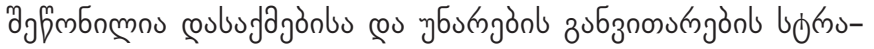

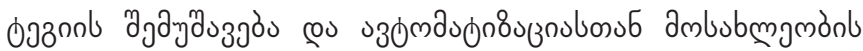

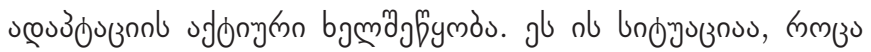

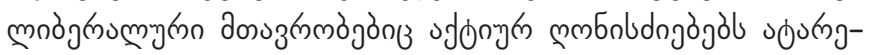

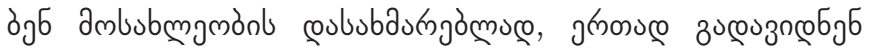

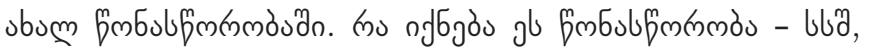

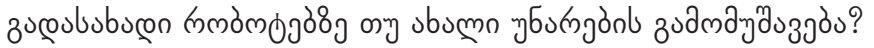

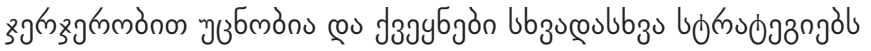

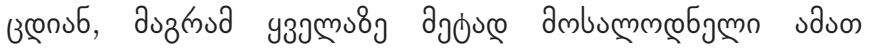

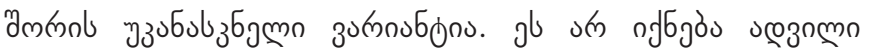

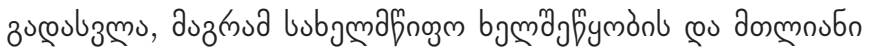

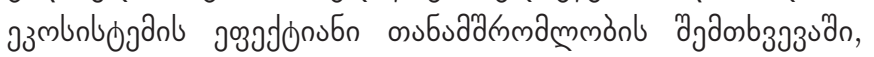

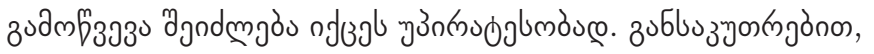

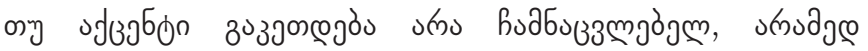

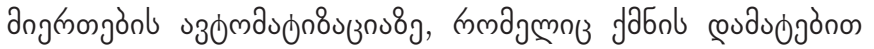

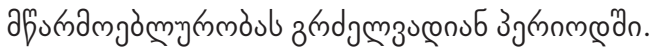

\section{@os335s}

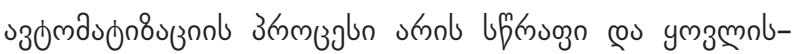

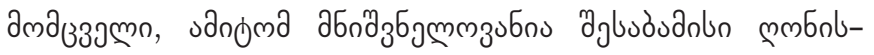

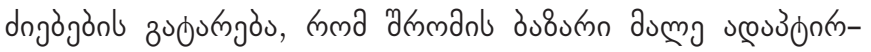

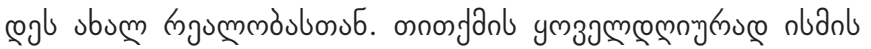

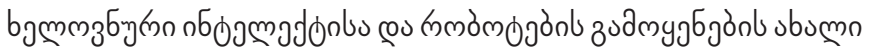

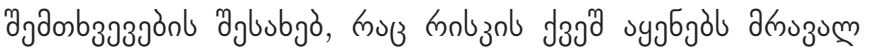

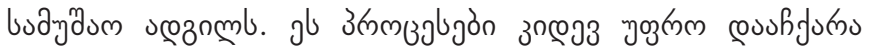

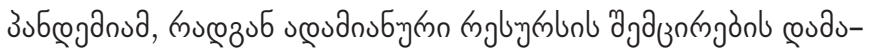

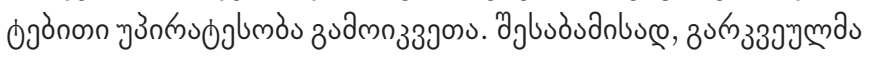

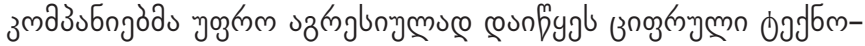
mmzngònl zudmyg

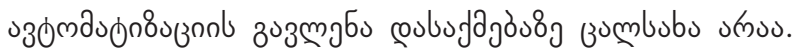

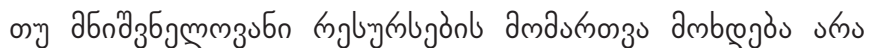

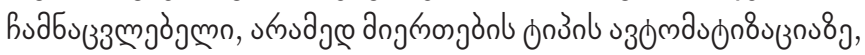

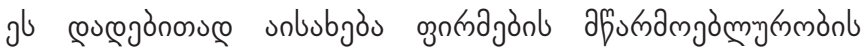

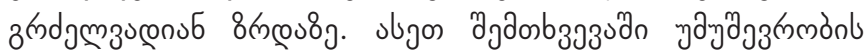

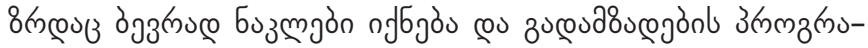

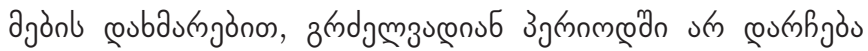

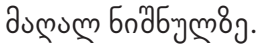

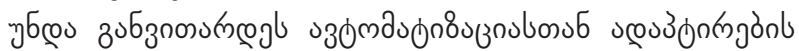

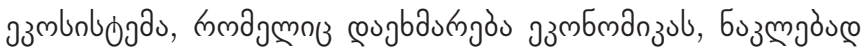

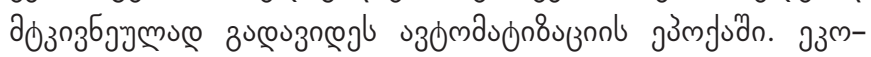

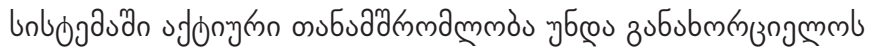

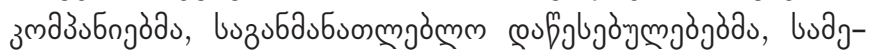

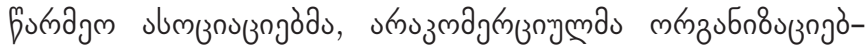

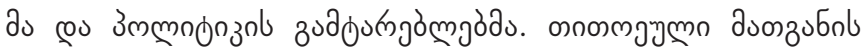

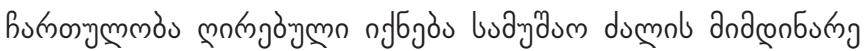

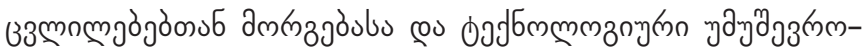

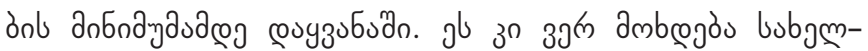

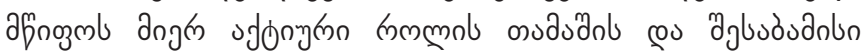

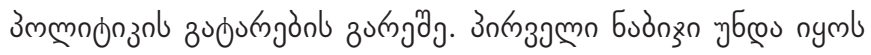

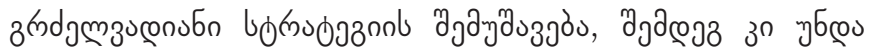

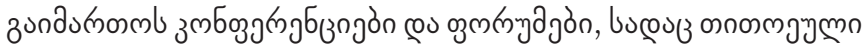

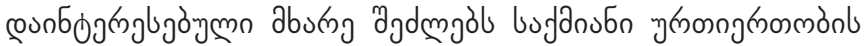

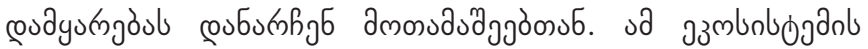

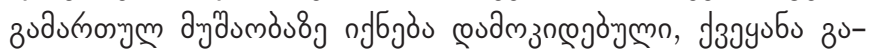

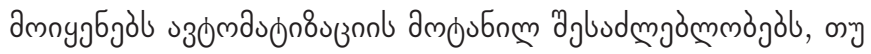

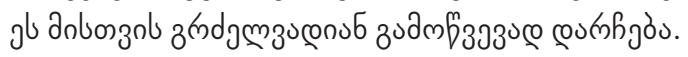

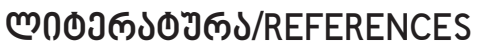

Bichia, Q. (2017). The effect of modern globalization and automatization trend on labor market. In: The 2nd International Scientific Conference: Challenges of Globalization in Economics and Business. Ivane Javakhishvili Tbilisi State University Press, Tbilisi, 96-102 (In Georgian)

Bedianashvili, G. (2018). Culture as a Factor of Knowledge Economics with Paradigmatic Changes in Systemic Institutional Context. Globalization and Business, N6, 58-66 (In Georgian)

Gagnidze, I. (2018). About Some Directions of the Formation of an Entrepreneurial Ecosystem in Georgia. The 3rd International Scientific Conference: Challenges of Globalization in Economics and Business, Tbilisi, 77-81 (In Georgian)

Gagnidze, I. (2019a). The Fourth Industrial Revolution - The Most Important Challenge of Higher Education in Georgia. Economics and Business, N3, 58-78 (In Georgian).

Gagnidze, I. (2019b). The Role of University Technology Transfer Offices in the Innovative Development of the Economy of Georgia. Globalization and Business, N8, 136-142 (In Georgian)

Gelashvili, M. (2018). Georgia is on the Path of Inclusive Growth. The 3rd International Scientific Conference: Challenges of Globalization in Economics and Business, Tbilisi, 82-85 (In Georgian)

Erkomaishvili, G. (2019). Digital Economy - an Innovative Tool for Economic Growth. Economics and Business, №2, 37-46 (In Georgian)

Tateishvili, A., \& Gugushvili, N. (2019). Automation of Processes in Customer Relations (Master Thesis) (In Georgian)

Abbott, R., \& Bogenschneider, B. (2018). Should robots pay taxes: Tax policy in the age of automation. Harvard Law \& Policy Review, 12(145) 
Acemoglu, D., \& Restrepo, P. (2018). Low-Skill and High-Skill Automation. Journal of Human Capital, 12(2), 204-232

Acemoglu, D., \& Restrepo, P. (2019). Automation and new tasks: how technology displaces and reinstates labor. Journal of Economic Perspectives, 33(2), 3-30

Acemoglu, D., \& Restrepo, P. (2020). Robots and jobs: Evidence from US labor markets. Journal of Political Economy, 128(6)

Baig, A., Hall, B., Jenkins, P., Lamarre, E. \& McCarthy, B. (May, 2020). The COVID-19 recovery will be digital: A plan for the first 90 days. McKinsey Global Institute

Bughin, J., Hazan, E., Lund, S., Dahlstrom, P., Wiesinger, A. \& Subramaniam, A. (2018). Skill shift: automation and the future of the workforce. McKinsey Global Institute

Chokheli, E. (2018). Strategy to Develop Fab Labs and Their Impact on Improving Business Activity in Georgia, World Science, Vol.2, No.6 (34), 4-8

Corkery, M. and Gelles, D. (2020). Robots Welcome to Take Over, as Pandemic Accelerates Automation. The New York Times

Frey, C. B., \& Osborne, M. A. (2017). The future of employment: How susceptible are jobs to computerisation? Technological Forecasting and Social Change, 114, 254-280

Moffitt, K. C., Rozario, A. M., \& Vasarhelyi, M. A. (2018). Robotic Process Automation for Auditing. Journal of Emerging Technologies in Accounting, 15(1), 1-10

OECD (2019), OECD Employment Outlook 2019: The Future of Work, OECD Publishing, Paris, https://doi.org/10.1787/9ee00155-en Raisch, S., \& Krakowski, S. (2020). Artificial Intelligence and Management: The Automation-Augmentation Paradox. Academy of Management Review, (ja)

Cornell University, INSEAD, and WIPO. (2019). The Global Innovation Index 2019.

World Economic Forum. (2016). The Future of Jobs: Employment, Skills and Workforce Strategy for the Fourth Industrial Revolution.

World Economic Forum. (2018). The Future of Jobs: Centre for the New Economy and Society.

https://knoema.com/atlas/ranks/RandD-expenditure?baseRegion=GE last accessed on 21.05.2020 